\title{
Neutrino masses from low scale partial compositeness
}

\author{
Zackaria Chacko, ${ }^{a}$ Patrick J. Fox, ${ }^{b}$ Roni Harnik ${ }^{b}$ and Zhen Liu ${ }^{a, c}$ \\ ${ }^{a}$ Maryland Center for Fundamental Physics, Department of Physics, \\ University of Maryland, College Park, MD 20742-4111, U.S.A. \\ ${ }^{b}$ Theoretical Physics Department, Fermilab, \\ P.O. Box 500, Batavia, IL 60510, U.S.A. \\ ${ }^{c}$ School of Physics and Astronomy, University of Minnesota, \\ Minneapolis, MN 55455, U.S.A. \\ E-mail: zchacko@umd.edu, pjfox@fnal.gov, roni@fnal.gov, \\ zliuphys@umn.edu
}

ABSTRACT: We consider a class of models in which the neutrinos acquire Majorana masses through mixing with singlet neutrinos that emerge as composite states of a strongly coupled hidden sector. In this framework, the light neutrinos are partially composite particles that obtain their masses through the inverse seesaw mechanism. We focus on the scenario in which the strong dynamics is approximately conformal in the ultraviolet, and the compositeness scale lies at or below the weak scale. The small parameters in the Lagrangian necessary to realize the observed neutrino masses can naturally arise as a consequence of the scaling dimensions of operators in the conformal field theory. We show that this class of models has interesting implications for a wide variety of experiments, including colliders and beam dumps, searches for lepton flavor violation and neutrinoless double beta decay, and cosmological observations. At colliders and beam dumps, this scenario can give rise to striking signals involving multiple displaced vertices. The exchange of hidden sector states can lead to observable rates for flavor violating processes such as $\mu \rightarrow e \gamma$ and $\mu \rightarrow e$ conversion. If the compositeness scale lies at or below a hundred $\mathrm{MeV}$, the rate for neutrinoless double beta decay is suppressed by form factors and may be reduced by an order of magnitude or more. The late decays of relic singlet neutrinos can give rise to spectral distortions in the cosmic microwave background that are large enough to be observed in future experiments.

Keywords: Beyond Standard Model, Neutrino Physics, Technicolor and Composite Models

ArXiv EPrint: 2012.01443 


\section{Contents}

1 Introduction 1

2 An inverse seesaw model from strong dynamics 5

3 Colliders 11

$3.1 W$ and $Z$ decays 12

3.2 Current constraints and future reach from HNL searches 16

$\begin{array}{lll}3.3 & \text { Novel signatures } & 19\end{array}$

4 Beam dumps $\quad 21$

5 Muon magnetic moment and lepton flavor violation 23

5.1 Muon magnetic moment 23

$\begin{array}{ll}5.2 \text { Lepton flavor violation } & 25\end{array}$

$6 \quad$ Neutrinoless double beta decay $\quad 27$

$\begin{array}{lll}7 & \text { Cosmology } & 30\end{array}$

7.1 Cosmological history 30

7.2 Cosmological bounds on the compositeness scale 32

7.3 Spectral distortions of the CMB from late decays 33

8 Astrophysics $\quad 34$

$\begin{array}{lll}9 & \text { Conclusions } & 37\end{array}$

$\begin{array}{ll}\text { A Matrix elements and widths } & 38\end{array}$

\section{Introduction}

Over the last few decades a series of experiments have conclusively established that neutrinos have tiny but non-vanishing masses and have determined their mass splittings. However, the mechanism that gives rise to these small masses remains a mystery. Among the many well-motivated proposals that have been put forward are the seesaw mechanism in its various different incarnations [1-13], and the Majoron model [14-16].

One attractive class of models that can naturally explain the smallness of the neutrino masses are those in which the neutrinos acquire mass through their couplings to the composite states of a strongly coupled sector [17-19]. Since the compositeness scale arises from dimensional transmutation, it can be parametrically lower than the Planck scale. The 


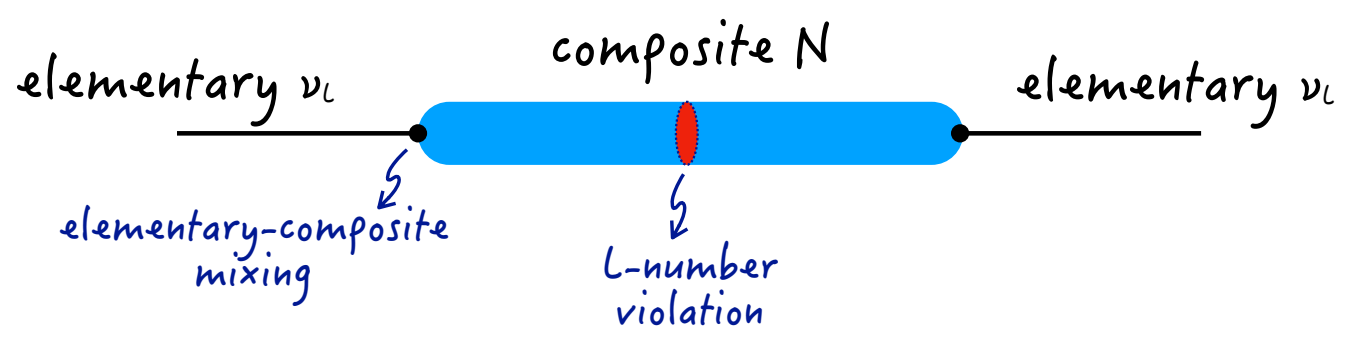

Figure 1. A sketch of the generation of neutrino masses in our framework through partial compositeness. Elementary neutrinos mix with composite singlet neutrinos and lepton number is violated in the composite sector. The light neutrino mass may be explained either by small mixing between the elementary and composite neutrinos, or by small lepton number violation in the composite sector, with a continuum of possibilities in between.

smallness of neutrino masses is explained by the fact they arise from operators of dimension greater than four. In this scenario the neutrinos could be either Dirac or Majorana particles. The composite neutrino framework has been linked to dark matter [20-22] and to the origin of the baryon asymmetry of the universe [23]. The cosmological signals of this class of theories have been considered in [24].

In this paper we explore a class of models that realize this scenario and show that they can lead to rich phenomenological consequences. We consider a framework in which the neutrinos acquire Majorana masses through mixing with singlet neutrinos that emerge as composite states of a strongly coupled hidden sector, as shown schematically in figure 1 . From the perspective of the low energy theory, at or below the compositeness scale, the neutrino masses arise through the inverse seesaw mechanism $[25,26]$. The difference between our framework and that of a conventional inverse seesaw is that the singlet neutrinos are now the hadrons of a new strong force, and the light neutrinos are partially composite particles. Our approach is similar to that of composite Higgs models, (for reviews see $[27,28])$, in which the SM fermions receive their masses through partial compositeness $[29,30]$. The composite singlet neutrinos are analogous to the vector-like fermion resonances in composite Higgs models. However here, in contrast to composite Higgs models, the strongly coupled sector is neutral under the SM gauge groups and so we are free to consider compositeness scales that lie well below the electroweak scale.

In this paper we focus on the scenario in which the compositeness scale, which we denote by $\Lambda$, lies at or below the weak scale. This allows the possibility of directly producing composite states at colliders and other experiments. For concreteness we take the strongly coupled hidden sector to be a conformal field theory (CFT). The conformal symmetry is broken at the scale $\Lambda$. Lepton number is also broken by dynamics in the hidden sector. The small parameters required to obtain the tiny neutrino masses within the framework of a low scale seesaw model can be understood here as naturally arising from the scaling dimensions of operators in the CFT.

The spectrum of the neutrino sector consists of the light neutrinos, which are now partially composite and parametrically lighter than the compositeness scale, and an infinite tower of composite neutrinos of which the lightest states have a mass of order the 


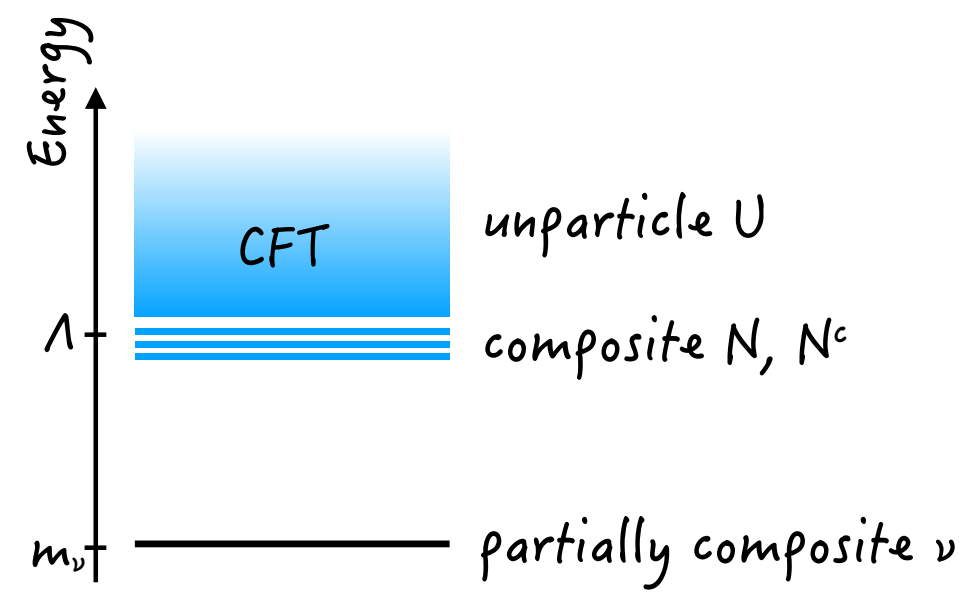

Figure 2. A sketch of the spectrum of particle masses in our framework, showing the light partially composite neutrinos and the composite resonances at the compositeness scale $\Lambda$ that blend into a continuum of "unparticles" at higher scales.

compositeness scale $\Lambda$. At scales well above $\Lambda$ the spectrum of composite states blends into a continuum spectrum of "unparticles" [31,32]. The spectrum of particle masses in this scenario is shown in figure 2.

The AdS/CFT correspondence [33-36] relates strongly coupled CFTs to theories of gravity in higher dimensions. Theories of phenomenological interest in which the strong conformal dynamics is spontaneously broken are dual [37, 38] to the two-brane RandallSundrum (RS) construction [39], originally proposed as a solution to the hierarchy problem of the SM. Several authors have explored neutrino masses within the framework of the RS solution to the hierarchy problem, for example [40-43]. It was shown in [44] that the AdS/CFT correspondence relates a certain class of these extra dimensional models to theories in which the neutrino masses are generated by the inverse seesaw mechanism, with composite states playing the role of the singlet neutrinos. These theories therefore share many features of our construction. However, an important difference is that in these theories the SM leptons are themselves partially or entirely composite. The compositeness scale is then constrained to lie above a $\mathrm{TeV}$, and consequently the phenomenology is very different. A holographic model of neutrino masses from low scale compositeness that is more closely related to our construction was considered in [45] (see also [46]).

The class of models that we are exploring has interesting implications for a wide variety of current and near-future experiments, including colliders and beam dumps, searches for lepton flavor violation and neutrinoless double beta decay, as well as cosmological observations. The range of compositeness scales that can be probed by each of these different types of signals is summarized in figure 3 . We now consider each of these different experimental probes in turn, along with the constraints from current data.

- LHC signals: this scenario can give rise to striking signals at colliders. A $W$ boson produced at the Large Hadron Collider (LHC) can now decay into a hard lepton and one or more composite singlet neutrinos. The singlet neutrinos subsequently decay 


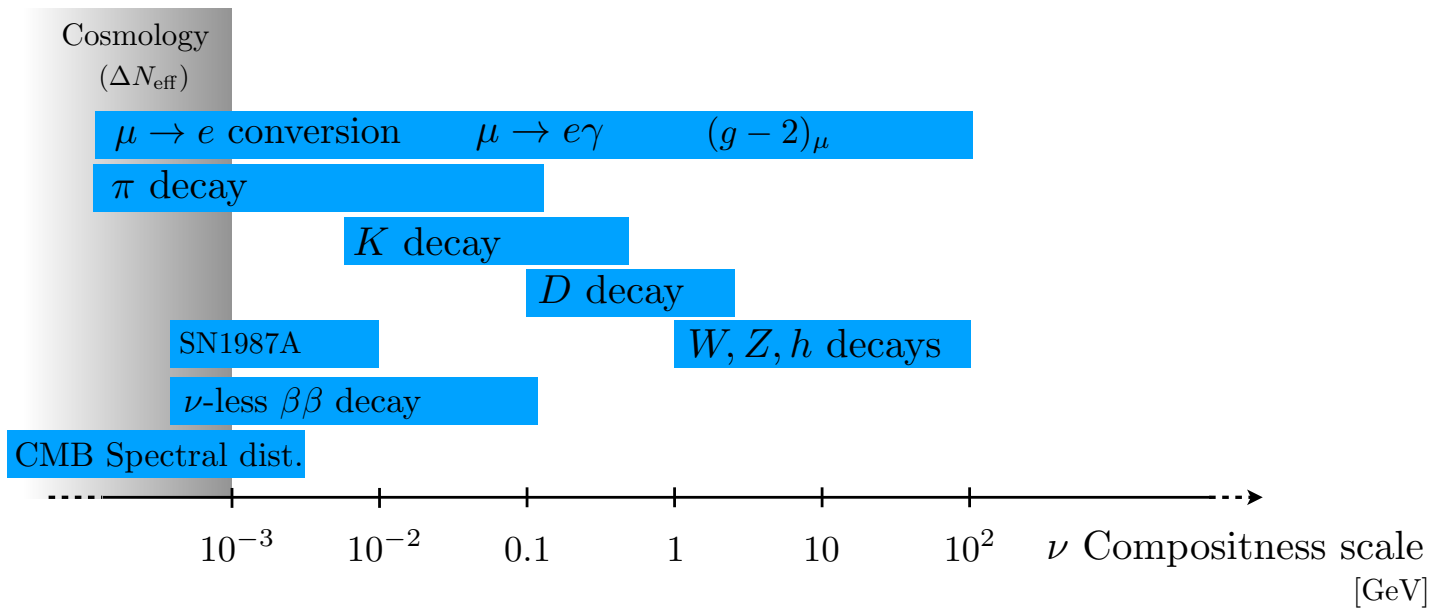

Figure 3. A sketch of the various probes of neutrino compositeness and the range of compositeness scales that they are potentially sensitive to.

into SM final states. In much of the parameter space, these decays are slow on collider timescales, giving rise to events with multiple displaced vertices. The observation of this striking signal would allow this class of theories to be distinguished from more conventional inverse seesaw models in which the singlet neutrinos are elementary. We find that the HL-LHC will have excellent sensitivity to this class of models. Codex-B, FASER-2, and MATHUSLA can all further improve on the HL-LHC reach. This is discussed in section 3 .

- Meson decays in beam dumps: if the singlet neutrino mass lies below a few $\mathrm{GeV}$, this class of models can also be discovered at beam dumps. Mesons produced at beam dumps can now decay into final states that contain one or more singlet neutrinos. The subsequent decays of these singlet neutrinos into SM final states can lead to striking signals, particularly in the case when these decays result in displaced vertices. We find that DUNE and SHiP are both sensitive to this scenario. This is discussed in further detail in section 4 .

- Charged lepton flavor violation: in general the couplings of the SM to the hidden sector violate the flavor symmetries of the lepton sector of the SM. Consequently, this scenario can give rise to lepton flavor violation at loop level through the exchange of hidden sector states. The COMET and Mu2e experiments, which are searching for the lepton flavor violating process $\mu \rightarrow e$ conversion, will be able to probe this class of models. We discuss this in detail in section 5 .

- Neutrinoless double $\beta$ decay: experiments searching for neutrinoless double beta decay are extremely important because the observation of this process would establish that neutrinos are Majorana particles. The characteristic momentum scale associated with neutrinoless double beta decay is set by the nucleon spacing in the nucleus, which is of order the pion mass. It follows that if the scale of compositeness in the 
neutrino sector lies at or below a hundred $\mathrm{MeV}$, the rate for neutrinoless double beta decay is suppressed by form factors and may be greatly reduced, with important consequences for experiment. We discuss this in detail in section 6 .

- Cosmology: in the early universe, thermal contact with the SM ensures that the hidden sector is populated. At temperatures below the compositeness scale the states in the hidden sector annihilate efficiently into light neutrinos, with the result that their abundance is exponentially suppressed. If the compositeness scale lies below an $\mathrm{MeV}$, the temperature at which the weak interactions decouple, this results in a correction to the total energy density in neutrinos that is unacceptably large. Values of $\Lambda$ below an $\mathrm{MeV}$ are therefore disfavored. The remaining relic composite states eventually decay into final states that, in addition to neutrinos, may also include charged particles. If the compositeness scale lies below $50 \mathrm{MeV}$, these late decays into charged states can give rise to spectral distortions in the cosmic microwave background (CMB) that are potentially observable in future experiments. The cosmological history of this class of models is discussed in section 7 .

- Supernovae: if the compositeness scale is lower than or of order $40 \mathrm{MeV}$, the temperature in the core of a supernova, hidden sector states can be produced at the core. Once produced, they get trapped inside the core and thermalize, and so they do not contribute to energy loss. The presence of these additional degrees of freedom would be expected to affect the supernova dynamics. However, given the present uncertainties in supernova dynamics we cannot robustly rule out this possibility. This is discussed in section 8 .

In the next section we present the framework that underlies this class of models and determine the allowed parameter space. In the subsequent sections we discuss each of the various types of signals as listed above. We conclude in section 9 .

\section{An inverse seesaw model from strong dynamics}

In this section we describe our framework for neutrino mass generation based on the partial compositeness of neutrinos. For concreteness, we will take the strong dynamics to be that of a conformal field theory (CFT) deformed by a relevant operator $\mathcal{O}_{\mathrm{S}}$. The corresponding terms in the Lagrangian take the form,

$$
\mathcal{L}_{\mathrm{UV}} \supset \mathcal{L}_{\mathrm{CFT}}+\lambda_{\mathrm{S}} \mathcal{O}_{\mathrm{S}}
$$

When the deformation $\mathcal{O}_{\mathrm{S}}$ gets large, it triggers breaking of the CFT at a scale which we denote by $\Lambda$. We focus on the scenario in which there are no pions or other light composite states, so that $\Lambda$ corresponds to the mass scale of the lightest composite particles. We require that the spectrum of light composite states contains at least two pairs of fermions $N$ and their Dirac partners $N^{c}$ that will play the role of singlet neutrinos. We will return to the issue of flavor at the end of this section, but for now we suppress all flavor indices. 
The low energy Lagrangian then includes kinetic terms and mass terms for the composite singlet neutrinos,

$$
\mathcal{L}_{\mathrm{IR}} \supset i \bar{N} \bar{\sigma}^{\mu} \partial_{\mu} N+i \bar{N}^{c} \bar{\sigma}^{\mu} \partial_{\mu} N^{c}-\left(M_{N} N^{c} N+\text { h.c. }\right)
$$

We expect that the mass parameter $M_{N}$ is of order $\Lambda$.

The CFT is assumed to couple to the SM through a neutrino portal interaction which, above the scale $\Lambda$, takes the form

$$
\mathcal{L}_{\mathrm{UV}} \supset \frac{\hat{\lambda}}{M_{\mathrm{UV}}^{\Delta_{\mathrm{N}}-3 / 2}} L H \mathcal{O}_{\mathrm{N}}+\text { h.c. } .
$$

In this expression the mass scale $M_{\mathrm{UV}}$ represents the ultraviolet cutoff of the theory, and $\hat{\lambda}$ is a dimensionless parameter taken to be $\mathcal{O}(1)$. Here $\mathcal{O}_{\mathrm{N}}$ is a primary operator of the CFT and $\Delta_{\mathrm{N}}$ is its scaling dimension. Unitarity places restrictions on the scaling dimensions of CFT primary operators which depend on their transformation properties under the Lorentz group. For spin $1 / 2$ operators such as $\mathcal{O}_{N}$, unitarity requires $\Delta_{N} \geq 3 / 2$. The limiting case of $\Delta_{N}=3 / 2$ corresponds to $\mathcal{O}_{\mathrm{N}}$ being a free fermion. For $\Delta_{\mathrm{N}} \geq 5 / 2$ the coupling in eq. (2.3) makes the theory ultraviolet sensitive, and additional counterterms involving the SM fields are required for consistency. We therefore focus on the range of scaling dimensions, $3 / 2 \leq \Delta_{\mathrm{N}}<5 / 2$. We use the two-point function to normalize the operator $\mathcal{O}_{\mathrm{N}}$, following the conventions of unparticle physics for fermionic operators [47],

$$
\int d^{4} x e^{i p x}\left\langle 0\left|T\left[\mathcal{O}_{\mathrm{N}}(x) \mathcal{O}_{\mathrm{N}}^{\dagger}(0)\right]\right| 0\right\rangle=\frac{A_{\Delta_{\mathrm{N}}-1 / 2}}{2 i \cos \left(\Delta_{\mathrm{N}} \pi\right)} \frac{\sigma^{\mu} p_{\mu}}{\left(-p^{2}-i \epsilon\right)^{5 / 2-\Delta_{\mathrm{N}}}}
$$

where

$$
A_{\Delta_{\mathrm{N}}-1 / 2}=\frac{16 \pi^{5 / 2}}{(2 \pi)^{2 \Delta_{\mathrm{N}}-1}} \frac{\Gamma\left(\Delta_{\mathrm{N}}\right)}{\Gamma\left(\Delta_{\mathrm{N}}-3 / 2\right) \Gamma\left(2 \Delta_{\mathrm{N}}-1\right)} .
$$

At energies of order $\Lambda$, the interaction in eq. (2.3) gives rise to a term in the low-energy Lagrangian of the form,

$$
\mathcal{L}_{\mathrm{IR}} \supset \lambda L H N+\text { h.c. }
$$

Based on our normalization of $\mathcal{O}_{\mathrm{N}}$, using the methods of "naive dimensional analysis" (NDA) [48-50] we estimate $\lambda$ to be of order,

$$
\lambda \sim C_{\lambda} \hat{\lambda}\left(\frac{\Lambda}{M_{\mathrm{UV}}}\right)^{\Delta_{\mathrm{N}}-3 / 2}
$$

where the order one multiplicative factor $C_{\lambda}$ is given by,

$$
C_{\lambda}=\frac{(4 \pi)^{3 / 2-\Delta_{\mathrm{N}}}}{\Gamma\left(\Delta_{\mathrm{N}}-3 / 2\right)} \sqrt{\frac{\pi}{\left(\Delta_{\mathrm{N}}-3 / 2\right) \cos \left(\Delta_{\mathrm{N}} \pi\right)}}
$$

The terms in eq. (2.2) and eq. (2.6) respect a global lepton number symmetry under which $L$ and $N^{c}$ carry charge +1 and $N$ has charge -1 . We now assume that at high energies, in 
addition to the terms in eq. (2.1) and eq. (2.3), the Lagrangian contains a small deformation of the CFT, denoted by $\mathcal{O}_{2 N^{c}}$, which explicitly violates lepton number,

$$
\mathcal{L}_{\mathrm{UV}} \supset \frac{\hat{\mu}^{c}}{M_{\mathrm{UV}}^{\Delta_{\mathrm{N}^{c}-4}}} \mathcal{O}_{2 \mathrm{~N}^{c}}+\text { h.c. }
$$

Here $\Delta_{2 \mathrm{~N}^{c}}$ is the scaling dimension of the operator $\mathcal{O}_{2 \mathrm{~N}^{c}}$, and $\hat{\mu}^{c}$ is a dimensionless parameter. The unitarity bound on the scaling dimensions of scalar operators restricts $\Delta_{2 \mathrm{~N}^{c}} \geq 1$, where the limiting case of $\Delta_{2 \mathrm{~N}^{c}}=1$ corresponds to a free scalar. For the range of scaling dimensions $1 \leq \Delta_{2 \mathrm{~N}^{\mathrm{c}}}<2$, we can normalize $\mathcal{O}_{2 \mathrm{~N}^{\mathrm{c}}}$ as per the conventions of unparticle physics, this time for scalar operators [31, 32],

$$
\int d^{4} x e^{i p x}\left\langle 0\left|T\left[\mathcal{O}_{2 \mathrm{~N}^{\mathrm{c}}}^{\dagger}(x) \mathcal{O}_{2 \mathrm{~N}^{\mathrm{c}}}(0)\right]\right| 0\right\rangle=-\frac{A_{\Delta_{2 \mathrm{~N}^{\mathrm{c}}}}}{2 i \sin \left(\Delta_{2 \mathrm{~N}^{\mathrm{c}}} \pi\right)} \frac{1}{\left(-p^{2}-i \epsilon\right)^{2-\Delta_{2 \mathrm{~N}^{\mathrm{c}}}}},
$$

The absorptive (imaginary) part of eq. (2.10) simplifies to [51],

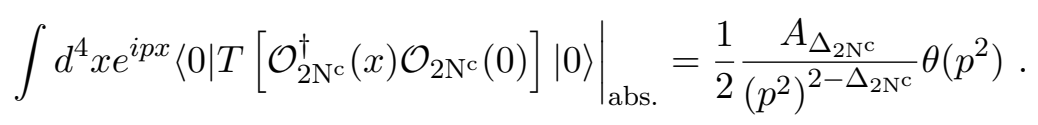

The operator normalization in eq. (2.10) is only valid for the range of scaling dimensions $1 \leq \Delta_{2 \mathrm{~N}^{\mathrm{c}}}<2$. For $\Delta_{2 \mathrm{~N}^{\mathrm{c}}} \geq 2$, the left-hand side of eq. (2.10) diverges in the ultraviolet, and we can no longer employ this normalization. However, the absorptive part of eq. (2.10), eq. (2.11), is ultraviolet safe. We therefore use eq. (2.11) to normalize the operator $\mathcal{O}_{2 \mathrm{~N}^{c}}$ over the entire range of scaling dimensions $\Delta_{2 \mathrm{~N}^{c}}>1$. Assuming this deformation carries a lepton number of $(-2)$, at scales of order $\Lambda$ the Lagrangian contains a lepton number violating term of the form,

$$
\mathcal{L}_{\mathrm{IR}} \supset \frac{\mu^{c}}{2}\left(N^{c}\right)^{2}+\text { h.c. }
$$

The mass parameter $\mu^{c}$ is related to the parameters in the ultraviolet theory as

$$
\mu^{c} \sim C_{\mu} \hat{\mu}^{c} \Lambda\left(\frac{\Lambda}{M_{\mathrm{UV}}}\right)^{\Delta_{2 \mathrm{~N}^{c}-4}},
$$

where the multiplicative factor $C_{\mu}$ is given by,

$$
C_{\mu}=\frac{(4 \pi)^{2-\Delta_{2 \mathrm{~N}^{\mathrm{c}}}}}{\Gamma\left(\Delta_{2 \mathrm{~N}^{\mathrm{c}}}-1\right)} \sqrt{\frac{1}{\Delta_{2 \mathrm{~N}^{c}}-1}} .
$$

We see from eqs. (2.2), (2.6) and (2.12) that the low energy Lagrangian contains all the ingredients necessary to realize the inverse seesaw,

$$
\mathcal{L}_{\mathrm{IR}} \supset i \bar{N} \bar{\sigma}^{\mu} \partial_{\mu} N+i \bar{N}^{c} \bar{\sigma}^{\mu} \partial_{\mu} N^{c}-\left[M_{N} N^{c} N+\lambda L H N+\frac{\mu^{c}}{2}\left(N^{c}\right)^{2}+\text { h.c. }\right] .
$$

Integrating out $N$ and $N^{c}$ we get a contribution to the masses of the light neutrinos from the inverse seesaw of order,

$$
\left.m_{\nu}\right|_{\text {inv.seesaw }}=\mu^{c}\left(\frac{\lambda v_{\mathrm{EW}}}{M_{N}}\right)^{2}
$$


with $v_{\mathrm{EW}} \approx 174 \mathrm{GeV}$. We expect comparable but slightly smaller contributions to the neutrino masses from integrating out the higher mass singlet fermion resonances. Therefore our final expression for the masses of the light neutrinos takes the form,

$$
m_{\nu} \sim \mu^{c}\left(\frac{\lambda v_{\mathrm{EW}}}{M_{N}}\right)^{2} \sim \Lambda\left[C_{\mu} \hat{\mu}^{c}\left(\frac{\Lambda}{M_{\mathrm{UV}}}\right)^{\Delta_{2 \mathrm{~N}^{c}}-4}\right]\left[C_{\lambda} \hat{\lambda}\left(\frac{v_{\mathrm{EW}}}{\Lambda}\right)\left(\frac{\Lambda}{M_{\mathrm{UV}}}\right)^{\Delta_{\mathrm{N}}-3 / 2}\right]^{2}
$$

The first square bracket in eq. (2.17) controls the strength of lepton number violation in the composite sector. The second square bracket controls the degree to which the elementary SM neutrinos mix with the composite singlet neutrinos. Both of these effects must be present to generate Majorana neutrino masses. The lightness of the neutrino may be explained in this framework either by the approximate lepton number symmetry in the composite sector or by the smallness of elementary-composite mixing, with a continuum of possibilities in between. We see from eq. (2.17) that the sizes of these effects depend on the scaling dimensions of the operators $\mathcal{O}_{\mathrm{N}}$ and $\mathcal{O}_{2 \mathrm{~N}^{c}}$. Therefore, in this framework, the scaling dimensions of CFT operators can provide a simple and natural explanation for the smallness of neutrino masses.

In obtaining eq. (2.17), we have assumed that the contributions to the neutrino mass from scales of order $\Lambda$ dominate over the contributions from higher scales. In general, in the presence of the deformations eqs. (2.3) and (2.9), we expect an ultraviolet contribution to the Weinberg operator $(L H)^{2}$. The size of this effect is controlled by the CFT three-point function $\left\langle\mathcal{O}_{\mathrm{N}}(x) \mathcal{O}_{\mathrm{N}}(y) \mathcal{O}_{2 \mathrm{~N}^{\mathrm{c}}}(z)\right\rangle$. From dimensional considerations we see that the condition that there not be a contribution to $(L H)^{2}$ that diverges in the ultraviolet translates into a bound on the scaling dimensions of the operators $\mathcal{O}_{\mathrm{N}}$ and $\mathcal{O}_{2 \mathrm{~N}^{c}}, 2 \Delta_{\mathrm{N}}+\Delta_{2 \mathrm{~N}^{\mathrm{c}}} \leq 8$. We assume that this bound is satisfied, so that the dominant contribution to the neutrino masses arises from scales of order $\Lambda$, and is given by eq. (2.17).

Another restriction on the parameter space arises from the condition that the Majorana mass for the singlet neutrinos arising from eq. (2.12) cannot be larger than the compositeness scale, $\mu^{c} \lesssim \Lambda$. This translates into a lower bound on the coupling $\lambda$. There is also an upper bound on $\lambda$ from the condition that the Dirac mass for the neutrinos obtained from eq. (2.6) be less than the compositeness scale. From this it follows that $\lambda$ must lie in the range

$$
\frac{M_{N}}{v_{\mathrm{EW}}} \gtrsim \lambda \gtrsim \frac{\sqrt{m_{\nu} M_{N}}}{v_{\mathrm{EW}}} .
$$

In the limit when $\lambda$ sits at its lower bound in eq. (2.18), the operator $\mathcal{O}_{2 \mathrm{~N}^{c}}$ may be playing the role of the operator $\mathcal{O}_{\mathrm{S}}$ that triggers the breaking of conformal symmetry.

In general the deformation $\mathcal{O}_{2 \mathrm{Nc}}$ will also generate a lepton number violating mass term for $N$ at the scale $\Lambda$,

$$
\mathcal{L}_{\text {IR }} \supset \frac{\mu}{2} N^{2}+\text { h.c. }
$$

The parameter $\mu$ is expected to be of order $\mu^{c}$. This term, although very similar in form to the lepton number violating mass term for $N^{c}$ shown in eq. (2.12), does not contribute significantly to the neutrino masses. 
The Lagrangian at the scale $\Lambda$ is also expected to contain four-fermion interactions between the $N$ 's. These take the schematic form,

$$
\mathcal{L}_{\mathrm{IR}} \supset-\kappa \frac{\left(\bar{N} \sigma^{\mu} N\right)^{2}}{\Lambda^{2}}+\kappa^{\prime} \frac{\left(N^{c} N\right)^{2}}{\Lambda^{2}}+\ldots,
$$

where we have shown two such terms. These interactions respect the overall lepton number symmetry. The parameters $\kappa$ and $\kappa^{\prime}$ are expected to be of order $(4 \pi)^{2}$. These nonrenormalizable terms are characteristic of the composite nature of the singlet neutrinos. Although not required to realize the inverse seesaw, they play an important role in the phenomenology of this class of models, particularly in the context of astrophysics and cosmology.

To generate the mass splittings necessary for neutrino oscillations there must be at least two pairs of $N, N^{c}$ and in general there may be more. Reinstating the flavor index that we have so far suppressed, the singlet neutrinos come in pairs $N_{\alpha}$ and $N_{\alpha}^{c}$ with $\alpha=$ $1,2, \ldots, \mathcal{N}_{N}$, with a corresponding number of operators $\mathcal{O}_{\mathrm{N}}^{\alpha}$ in the CFT. Including this flavor index the couplings between the SM lepton doublets and the CFT operators are now given by,

$$
\mathcal{L}_{\mathrm{UV}} \supset \frac{\hat{\lambda}_{i \alpha}}{M_{\mathrm{UV}}^{\Delta_{\mathrm{N}}-3 / 2}} L^{i} H \mathcal{O}_{\mathrm{N}}^{\alpha}+\text { h.c. . }
$$

The flavor index on the lepton doublets runs over the SM generations, $i=1,2,3$. We work in the basis in which the charged lepton masses are flavor diagonal.

Keeping track of flavor indices, the low energy Lagrangian in eq. (2.15) now takes th form,

$$
\begin{aligned}
\mathcal{L}_{\mathrm{IR}} \supset & i \bar{N}_{\alpha} \bar{\sigma}^{\mu} \partial_{\mu} N_{\alpha}+i \bar{N}_{\alpha}^{c} \bar{\sigma}^{\mu} \partial_{\mu} N_{\alpha}^{c} \\
& -\left[\left(M_{N}\right)_{\alpha \beta} N_{\alpha}^{c} N_{\beta}+\lambda_{i \alpha} L^{i} H N^{\alpha}+\frac{\left(\mu^{c}\right)_{\alpha \beta}}{2} N_{\alpha}^{c} N_{\beta}^{c}+\text { h.c. }\right]
\end{aligned}
$$

The $N_{\alpha}, N_{\alpha}^{c}$ may be rotated to make $M_{N}$ diagonal. In this basis, the SM neutrinos will, in general, couple to all the $N_{\alpha}$. We therefore expect that there will be both flavor preserving and flavor violating processes involving the heavy neutrinos. The mixing between the neutrinos is conventionally parametrized in terms of a mixing matrix $U$. In the limit of small mixing between the active and the composite singlet neutrinos, the corresponding elements of the mixing matrix are related to the parameters in eq. (2.22) as,

$$
U_{N_{\alpha} \ell_{i}}=\frac{\lambda_{i \alpha} v_{\mathrm{EW}}}{M_{N_{\alpha}}} .
$$

In our study of the phenomenology of this class of models, we will make certain simplifying assumptions. We assume that there is a discrete symmetry in the CFT that relates the different $\mathcal{O}_{\mathrm{N}}^{\alpha}$ operators so that they all have the same scaling dimension, $\Delta_{\mathrm{N}}$. We further assume that this symmetry is preserved in the breaking of conformal invariance by the operator $\mathcal{O}_{\mathrm{S}}$, so that all the composite singlet neutrinos are approximately degenerate. For concreteness, we assume that the $N_{\alpha}$ and $N_{\alpha}^{c}$ are the lightest states in the hidden sector, so that their only kinematically allowed decay modes are to SM particles. In order to relate 


\begin{tabular}{|c|cccccc|ccc|}
\hline & $\hat{\lambda}$ & $\hat{\mu}^{c}$ & $\Delta_{\mathrm{N}}$ & $\Delta_{2 \mathrm{~N}^{c}}$ & $\Lambda[\mathrm{MeV}]$ & $M_{\mathrm{UV}}[\mathrm{TeV}]$ & $\lambda$ & $\mu^{c}[\mathrm{MeV}]$ & $m_{\nu}[\mathrm{eV}]$ \\
\hline $\mathrm{I}$ & 1 & 1 & 1.85 & 3.9 & 400 & $M_{\mathrm{Pl}}$ & $8 \times 10^{-8}$ & 80 & 0.1 \\
$\mathrm{II}$ & 1 & 2 & 1.8 & 4.05 & 40 & $M_{\mathrm{Pl}}$ & $4 \times 10^{-7}$ & $10^{-2}$ & 0.045 \\
\hline $\mathrm{III}$ & $5 \times 10^{-5}$ & 1 & 1.9 & 3.8 & 40 & $2 \times 10^{3}$ & $2 \times 10^{-8}$ & 7 & 0.05 \\
$\mathrm{IV}$ & 1 & $4 \times 10^{-14}$ & 2.4 & 2.25 & 400 & $2 \times 10^{3}$ & $3 \times 10^{-7}$ & 5 & 0.08 \\
$\mathrm{~V}$ & $10^{-2}$ & $5 \times 10^{-3}$ & 2.25 & 3.4 & 400 & $2 \times 10^{3}$ & $3 \times 10^{-8}$ & 300 & 0.05 \\
\hline
\end{tabular}

Table 1. Some representative choices of parameters that lead to neutrino masses of parametrically the right size. Shown are the dimensionless couplings $\hat{\lambda}$ and $\hat{\mu}^{c}$, the scaling dimensions $\Delta_{\mathrm{N}}$ and $\Delta_{2 \mathrm{~N}^{c}}$, the compositeness scale $\Lambda$, and the cutoff scale $M_{\mathrm{UV}}$. Also shown are some derived parameters, in particular the coupling constant $\lambda$, the Majorana mass term for the composite singlet neutrinos $\mu^{c}$, and the resulting mass scale of the light neutrinos. For each set of parameters all the constraints outlined in the paper are satisfied. In the upper set of benchmarks, the smallness of the neutrino masses is fully explained by the running of parameters from the Planck scale cutoff down to the infrared. In the lower set of benchmarks, the ultraviolet cutoff is lowered to $2 \mathrm{PeV}$, and the light masses of the neutrinos are explained, at least in part, by symmetries.

measurements performed at energies above the compositeness scale to the parameters in the low energy theory, we will take eqs. (2.7), (2.13) and (2.17) to be exact equalities rather than estimates. Then, although the bounds and projections we obtain are only accurate up to $\mathcal{O}(1)$ factors, this should suffice to give a sense of the current constraints on this class of models and the expected reach of future experiments.

In table 1, we present a few benchmark sets of model parameters that lead to neutrino masses in the right range. We will refer to the benchmarks as I-V in the order that they appear in the table. All the benchmark models have compositeness scales that lie well below the electroweak scale. The benchmarks fall into two broad categories that provide different explanations for the smallness of neutrino masses. In the first set of benchmarks, the parameters $\hat{\lambda}$ and $\hat{\mu}^{c}$ are order one at $M_{\mathrm{UV}}$. The smallness of neutrino masses is explained by the scaling dimensions of the operators $\mathcal{O}_{\mathrm{N}}$ and $\mathcal{O}_{2 \mathrm{~N}^{\mathrm{c}}}$, which control the extent to which the SM neutrinos mix with their composite counterparts and the extent of overall lepton number violation respectively. In the second set of benchmarks, the smallness of neutrino masses is explained, in part, by approximate global symmetries of the theory in the ultraviolet. There are two symmetries that can play a role here. In the limit that $\hat{\mu}^{c}$ vanishes, the theory has an exact lepton number symmetry. In the limit that $\hat{\lambda}$ vanishes, the SM decouples from the hidden sector and the lepton number symmetry of the SM is restored. Hence approximate symmetries can explain why either $\hat{\mu}^{c}$ or $\hat{\lambda}$ is small and thereby provide an explanation for the smallness of neutrino masses.

In the first set of benchmarks the cutoff $M_{\mathrm{UV}}$ is taken to very high, of order the Planck scale, $M_{\mathrm{Pl}}=1.2 \times 10^{19} \mathrm{GeV}$. The dimensionless couplings $\hat{\lambda}$ and $\hat{\mu}$ are of order one, with the small parameters necessary to realize the scale seesaw mechanism at low scales arising from renormalization group evolution from the Planck scale down to the compositeness scale $\Lambda$. In this scenario, even small modifications to the scaling dimensions of the operators $\mathcal{O}_{\mathrm{N}}$ and $\mathcal{O}_{2 \mathrm{~N}^{c}}$ can lead to large changes in the values of the infrared parameters. It is instructive to 
track the size of overall lepton number violation, which is set by $\hat{\mu}^{c}$ in the ultraviolet and by $\mu^{c} / \Lambda$ at the compositeness scale, and the size of SM lepton number violation, which is set by $\hat{\lambda}$ in the ultraviolet and by $\lambda v_{\mathrm{EW}} / M_{N}$ at the compositeness scale. Within this class of models, both these symmetries are violated by order one at the Planck scale, in line with the expectation that quantum gravity violates all global symmetries. The tight experimental constraints on mixing between SM neutrinos and singlet neutrinos imply that SM lepton number must necessarily be a symmetry to very good accuracy at low energies. This tends to naturally occur in our framework since the interaction $L H \mathcal{O}_{\mathrm{N}}$ that generates this mixing is always irrelevant. For high values of $M_{\mathrm{UV}}$, even if $\hat{\lambda}$ is of order one in the ultraviolet, the mixing can easily be sufficiently suppressed so as to result in suitably small masses for the light neutrinos. This remains true even if $\mathcal{O}_{2 \mathrm{~N}^{c}}$ is a (slightly) relevant operator, so that overall lepton number is violated by order one in the infrared, as illustrated in Benchmark I in the table. However, if $\mathcal{O}_{2 N^{c}}$ is instead a (slightly) irrelevant operator, overall lepton number can also emerge as an accidental symmetry at the compositeness scale, in which case it will also play a role in the suppression of neutrino masses. This is illustrated in Benchmark II in the table.

In the second set of benchmarks we take $M_{\mathrm{UV}}$ to be low, of order the flavor scale, $2 \times 10^{3} \mathrm{TeV}$. There is now less room for renormalization group evolution to generate the small parameters necessary to accommodate a low scale seesaw model. Therefore, in this scenario, symmetries are also expected to play a role in suppressing the neutrino masses. Neutrino masses in the right range can be obtained if $\hat{\lambda}$ is very small at $M_{\mathrm{UV}}$, so that SM lepton number is a symmetry to very good accuracy in the ultraviolet. This can happen even if overall lepton number is violated by order one in the infrared, as illustrated in Benchmark III in table 1. Alternatively, a realistic spectrum of neutrino masses can be obtained if overall lepton number is a symmetry to very good accuracy at $M_{\mathrm{UV}}$, even if SM lepton number is violated by order one at that scale. This is illustrated in Benchmark IV. It is also possible that both $\hat{\mu}^{c}$ and $\hat{\lambda}$ are small, so that both overall lepton number and SM lepton number are only mildly violated at the cutoff scale, as in Benchmark V.

It is important to note that the symmetries at $M_{\mathrm{UV}}$ in the low scale models could naturally emerge from dynamical effects. For example, one could easily imagine a different CFT above $M_{\mathrm{UV}}$ that serves as an ultraviolet completion for the low scale models, and for which the scaling dimensions are such that these symmetries emerge at low energies. This CFT could be broken at $M_{\mathrm{UV}}$ resulting in low scale theories with the features we present in the table, in which SM lepton number, hidden sector lepton number, or both, are approximate symmetries.

In summary, our framework naturally allows for the generation of the small couplings required to realize the inverse seesaw mechanism at low scales, and a wide range of low energy parameters are possible. In the following sections we will connect the parameter space to specific experimental observations.

\section{Colliders}

In this section we discuss the phenomenology of this class of models at colliders. We focus on the case in which the composite singlet neutrinos have masses at or below the weak scale. 


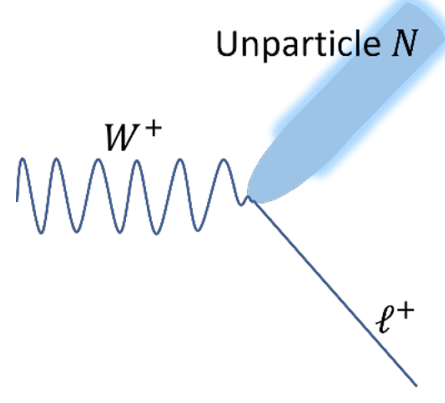

Figure 4. Unparticle production from $W$ boson decays.

We begin with a concrete and detailed calculation of the decay rate of $W$ and $Z$ gauge bosons into composite singlet neutrinos. We then translate the existing constraints from searches for elementary heavy neutral leptons (HNLs) to determine the range of parameter space that is currently allowed by the data. We also determine the reach of similar searches in the future. Finally, we sketch the novel collider signatures of this class of models, which include $W$ decays that give rise to multiple displaced vertices, and discuss the prospects of the HL-LHC for these signals.

\section{1 $W$ and $Z$ decays}

The hidden sector interacts with the SM through the neutrino portal interaction, eq. (2.3). Hidden sector states can therefore be produced in decays of the $W^{ \pm}$and $Z$ bosons through diagrams involving an off-shell neutrino. After being produced, the hidden sector hadronizes into final states consisting of one or more composite singlet neutrinos. In much of parameter space the composite singlet neutrinos are unstable on detector timescales and so the search is for their decay products.

We first consider the inclusive decay of a $W$ boson into a single flavor of lepton and unparticles, $W^{ \pm} \rightarrow \ell^{ \pm}+\mathcal{U}$, as shown in figure 4 . The leading order matrix element contains a single insertion of the interaction eq. (2.3),

$$
i \mathcal{M}=\frac{i g}{2 \sqrt{2}} \bar{u}\left(p_{N}\right)\left(\frac{-i \hat{\lambda} v_{\mathrm{EW}}}{M_{\mathrm{UV}}^{\Delta_{\mathrm{N}}-3 / 2}}\right) \frac{i \not p_{N}}{p_{N}^{2}} \gamma^{\mu}\left(1-\gamma^{5}\right) v\left(p_{\ell}\right) \epsilon_{\mu}\left(p_{W}\right) .
$$

Here $g$ is the $\mathrm{SU}(2)_{\mathrm{L}}$ gauge coupling while $p_{W}$ represents the four-momentum of the decaying $W$ boson, $p_{\ell}$ the four-momentum of the charged lepton, and $p_{N}=p_{W}-p_{\ell}$ the momentum carried away by the unparticles. In the rest frame of the decaying $W$ the spin averaged matrix element, after summing over the spins of the final state particles, is given by,

$$
\frac{1}{3} \sum|\mathcal{M}|^{2}=\frac{g^{2}}{3} \frac{\hat{\lambda}^{2} v_{\mathrm{EW}}^{2}}{M_{\mathrm{UV}}^{2 \Delta_{N}-3}} \frac{E_{\ell}}{m_{W}} \frac{3 m_{W}-2 E_{\ell}}{m_{W}-2 E_{\ell}} .
$$

Here $E_{\ell}$ represents the energy of final state charged lepton. The partial decay width is determined by integrating over the phase space densities of the charged lepton and the 
unparticles,

$$
d \Gamma=\frac{|\mathcal{M}|^{2}}{2 m_{W}}(2 \pi)^{4} \delta^{(4)}\left(p_{W}-p_{\ell}-p_{N}\right) d \Phi_{\ell} d \Phi_{\mathcal{O}} .
$$

The phase spaces of the charged lepton and the unparticles are given by

$$
d \Phi_{\ell}=\frac{1}{(2 \pi)^{3}} \frac{1}{2 E_{\ell}} d^{3} p_{\ell}, \quad d \Phi_{\mathcal{O}}=\frac{A_{\Delta_{N}-1 / 2}}{(2 \pi)^{4}} \theta\left(p_{N}^{0}\right) \theta\left(p_{N}^{2}-\mu_{\mathrm{IR}}^{2}\right)\left(p_{N}^{2}-\mu_{\mathrm{IR}}^{2}\right)^{\Delta_{\mathrm{N}}-5 / 2} d^{4} p_{N} .
$$

The expression for $A_{n}$, the phase space volume factor, was given in eq. (2.5). In the expression for the unparticle phase space we have introduced an infrared cutoff, $\mu_{\mathrm{IR}}$, to account for the fact that conformal invariance is broken at the compositeness scale. Performing the integration over the angular variables we obtain an expression for the energy distribution of the final state lepton,

$$
\begin{aligned}
\frac{d \Gamma}{d E_{\ell}}= & \frac{g^{2}}{96 \pi^{2}} \frac{\left|\hat{\lambda} v_{\mathrm{EW}}\right|^{2}}{m_{W}^{2}}\left(\frac{m_{W}}{M_{\mathrm{UV}}}\right)^{2 \Delta_{\mathrm{N}}-3} A_{\Delta_{\mathrm{N}}-1 / 2}\left(\frac{3 m_{W}-2 E_{\ell}}{m_{W}-2 E_{\ell}}\right) \\
& \times\left(\frac{2 E_{\ell}}{m_{W}}\right)^{2}\left(1-\frac{2 E_{\ell}}{m_{W}}-\frac{\mu_{\mathrm{IR}}^{2}}{m_{W}^{2}}\right)^{\Delta_{\mathrm{N}}-5 / 2} .
\end{aligned}
$$

For $\Delta_{N}<5 / 2$ the integral over $E_{\ell}$ for the total width is infrared divergent, with the divergence arising from the neighborhood of $E_{\ell}=m_{W} / 2$. Physically, this divergence is regulated by the fact that the conformal symmetry is broken and so the final state particles in the unparticle sector, the composite singlet neutrinos, are not massless but have masses of order the compositeness scale $\Lambda$. Therefore the point $E_{\ell}=m_{W} / 2$ can never actually be reached. By choosing a nonzero value of $\mu_{\mathrm{IR}}^{2}$ we regulate this divergence. In practice, there is an additional cutoff arising from the requirement that the decay products of $N$ be sufficiently energetic to be observed in the collider environment. The partial width is then given by,

$$
\Gamma(W \rightarrow \ell \mathcal{U})=m_{W} \frac{|\hat{\lambda}|^{2}}{48 \pi^{2}}\left(\frac{m_{W}}{M_{\mathrm{UV}}}\right)^{2 \Delta_{\mathrm{N}}-3} A_{\Delta_{\mathrm{N}}-1 / 2} f\left(\Delta_{\mathrm{N}}, \frac{\mu_{\mathrm{IR}}^{2}}{m_{W}^{2}}\right)
$$

The function $f\left(\Delta_{\mathrm{N}}, \mu_{\mathrm{IR}}^{2} / m_{W}^{2}\right)$ captures the dependence on the scaling dimension $\Delta_{\mathrm{N}}$ and $\mu_{\mathrm{IR}}$. Its detailed form is given in eq. (A.7) of the appendix. After rewriting the $W$ partial width as a function of the infrared parameters, it can be expressed in terms of the conventional HNL mixing angle using eqs. (2.7) and (2.23),

$$
\Gamma(W \rightarrow \ell \mathcal{U})=\sum_{\alpha=1}^{\mathcal{N}_{N}} m_{W} \frac{g^{2}\left|U_{N_{\alpha}}\right|^{2}}{96 \pi^{2} C_{\lambda}^{2}}\left(\frac{M_{N}}{m_{W}}\right)^{2}\left(\frac{m_{W}}{\Lambda}\right)^{2 \Delta_{N}-3} A_{\Delta_{N}-1 / 2} f\left(\Delta_{\mathrm{N}}, \frac{\mu_{\mathrm{IR}}^{2}}{m_{W}^{2}}\right) .
$$

Since the final result is sensitive to the value of $\mu_{\mathrm{IR}}^{2}$, this expression must be considered as just an estimate. As a consistency check we consider the limit that $\Delta_{\mathrm{N}} \rightarrow 3 / 2$,

$$
\lim _{\Delta_{\mathrm{N}} \rightarrow 3 / 2} A_{\Delta_{\mathrm{N}}-1 / 2} f\left(\Delta_{\mathrm{N}}, \mu_{\mathrm{IR}}\right)=\frac{2 \pi\left(\mu_{\mathrm{IR}}^{6}-3 \mu_{\mathrm{IR}}^{2} m_{W}^{4}+2 m_{W}^{6}\right)}{\mu_{\mathrm{IR}}^{2} m_{W}^{4}} .
$$




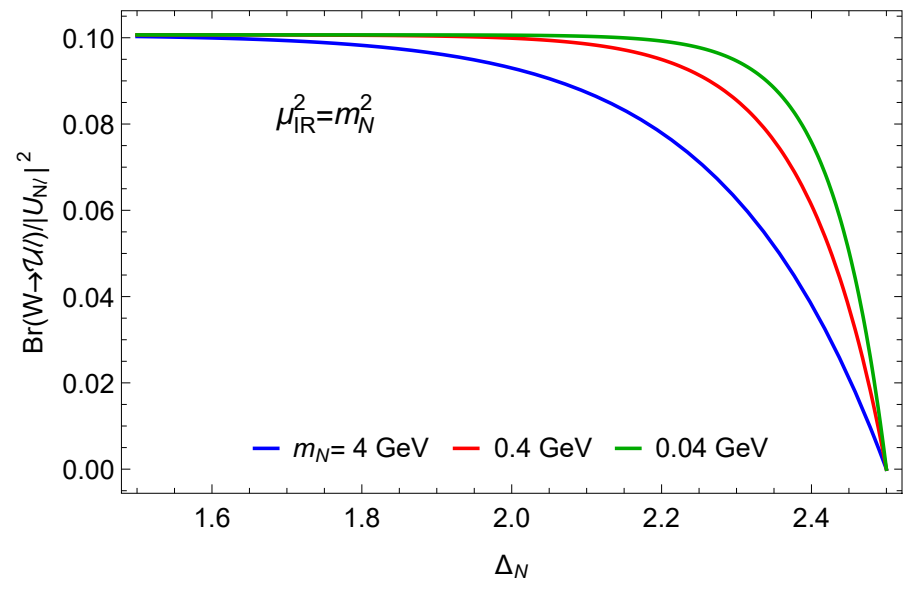

Figure 5. The branching fraction of $W \rightarrow \ell \mathcal{U}$, normalized by the IR mixing angle $\sum_{\alpha}\left|U_{N_{\alpha}}\right|^{2}$, for benchmark choices of $m_{N}$, as a function of the scaling dimension $\Delta_{N}$.
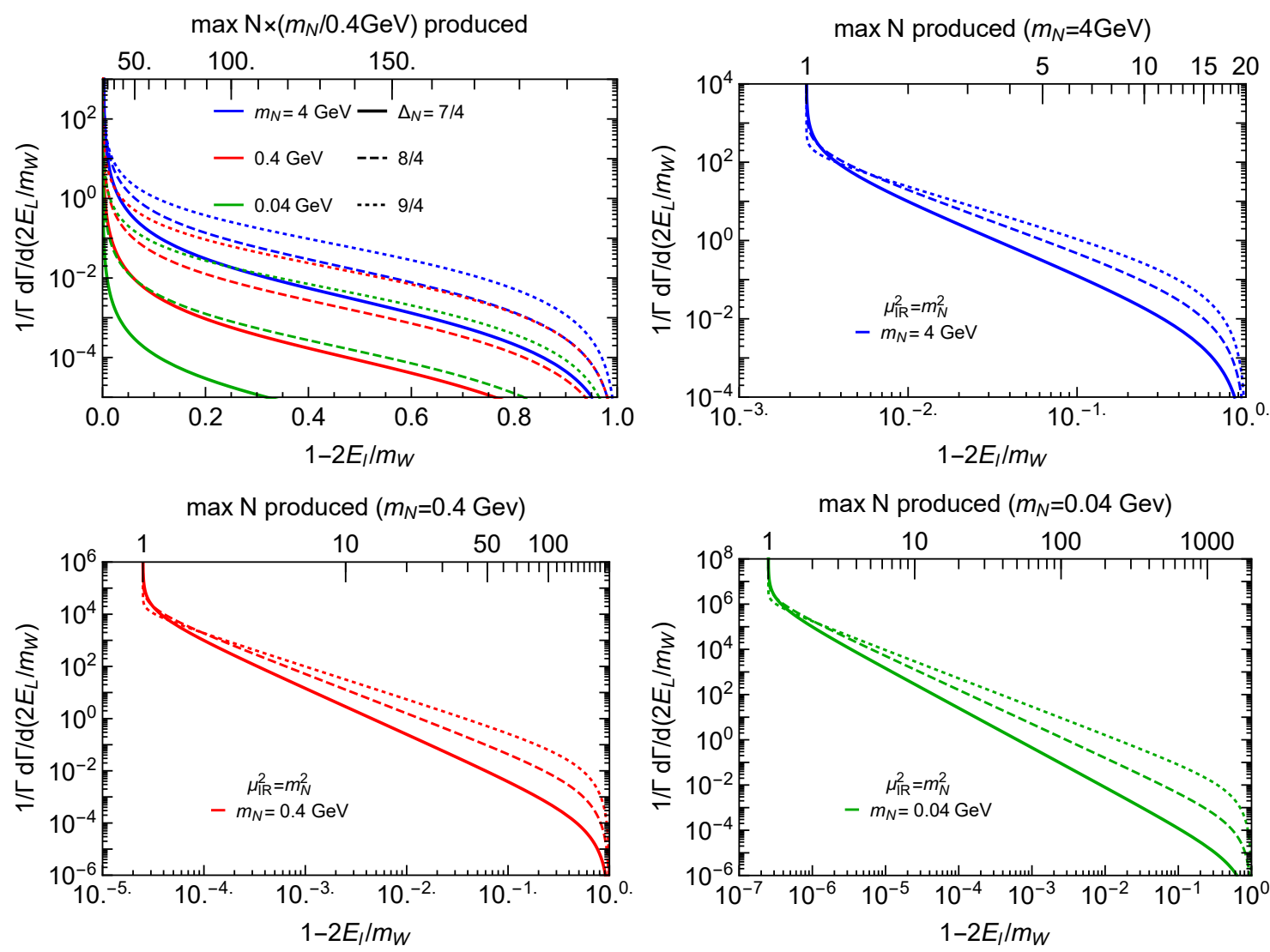

Figure 6. The differential width of $W \rightarrow \ell \mathcal{U}$ as a function of the charged lepton energy $E_{\ell}$ for various benchmark choices of $m_{N}$ and scaling dimension $\Delta_{N}$. The solid, dashed, and dotted lines represent benchmark scaling dimension of $\Delta_{\mathrm{N}}$ of $7 / 4,2$, and $9 / 4$, respectively. The green, red, and blue curves corresponds to singlet neutrino masses of $0.04,0.4$, and $4 \mathrm{GeV}$, respectively. On the upper axis of the figure we also show the maximum number of singlet neutrinos allowed by kinematics. The upper left panel shows the overall distribution in log-linear scale, while the rest of panels zoom in to near the maximum $E_{\ell}$ value and show the distribution in $\log -\log$ scale. 
In this limit, $C_{\lambda} \rightarrow 1$. Then, setting $\mu_{\mathrm{IR}}=m_{N}$, eq. (3.7) reproduces the standard result for an elementary HNL, as expected. Going forward, we shall set $\mu_{\mathrm{IR}}=m_{N}$ in this section when presenting results.

In figure 5 we show the branching fraction of $W \rightarrow \ell \mathcal{U}$, normalized by the infrared mixing angle $\sum\left|U_{N_{\alpha} \ell}\right|^{2}$, as a function of the scaling dimension $\Delta_{\mathrm{N}}$ for various benchmark choices of $M_{N}$. This branching fraction is sensitive to the choice of the infrared cutoff. The dependence on scaling dimensions, as a function of $\Delta_{N}$, is dominated by two effects, the increase from $1 / C_{\lambda}$ and the decrease with multi-body phase space of $A_{\Delta_{\mathrm{N}}-1 / 2} \times f\left(\Delta_{\mathrm{N}}, \mu_{\mathrm{IR}}^{2} / m_{W}^{2}\right)$. The multi-body phase space dominates the behavior and drives the decrease of the branching fractions into unparticle states.

So far we have discussed the inclusive rate for $W$ bosons to decay to unparticles. However, below the scale at which conformal symmetry is broken the unparticles must "hadronize" into singlet neutrinos so the ultimate fate of the decay is $W^{ \pm} \rightarrow \ell^{ \pm}+n N$, with $n$ an odd integer. The maximum number of final state singlet neutrinos $N$ that is kinematically allowed, for a given charged lepton energy $E_{\ell}$ in the $W$-boson center of mass frame, is given by,

$$
n_{\max }=\frac{m_{W}}{m_{N}} \sqrt{1-\frac{2 E_{\ell}}{m_{W}}} .
$$

In figure 6 we show the differential width of the $W$ to final states that include a charged lepton and an arbitrary number of singlet neutrinos as a function of the charged lepton energy. The form of these distributions is independent of the parameter $\hat{\lambda}$. The results are presented for three benchmark values of the scaling dimension, $\Delta_{N}=7 / 4,8 / 4$ and $9 / 4$, and for three benchmark masses of the composite singlet neutrinos, $M_{N}=0.04,0.4$, and $4 \mathrm{GeV}$. We omitted the case of $\Delta_{\mathrm{N}}=3 / 2$ since it is simply a delta function. In the top left panel, we show the overall distribution as a function of $E_{\ell}$, which is plotted on a linear scale, for the benchmark values of the masses and $\Delta_{\mathrm{N}}$. In the rest of the panels, we show the same distribution for each of the benchmark values of $M_{N}$ separately, but now with $E_{\ell}$ plotted on a logarithmic scale, zooming into the threshold region. From this figure we can see that the charged lepton energy favors the kinematic threshold of $E_{\ell} \simeq m_{W} / 2$, and is separated from it only by the non-vanishing mass of the HNL. Note that the range of the horizontal axis extends to different values of $E_{\ell}$ in these three panels, reflecting the different benchmark composite singlet neutrino masses. As a result of this behavior and the unitarity of the area under the differential curve, the heavier the composite singlet neutrinos, or equivalently the larger the infrared cut off $\mu_{\mathrm{IR}}$, the greater the fraction of events with charged lepton energies at lower values. Due to the shape of this distribution, the unparticle decays of $W$ bosons strongly favor low numbers of singlet neutrinos in the final state.

The rate for $Z$ boson decay into hidden sector states can be calculated in the same manner as for $W$ decay. The square of the resulting matrix element takes a form very similar to that for $W$ decay, eq. (3.2),

$$
\frac{1}{3} \sum|\mathcal{M}|^{2}=\frac{\left(g^{2}+g^{\prime 2}\right)}{6} \frac{\hat{\lambda}^{2} v_{\mathrm{EW}}^{2}}{M_{\mathrm{UV}}^{2 \Delta_{\mathrm{N}}-3}} \frac{E_{\ell}}{m_{Z}} \frac{3 m_{Z}-2 E_{\ell}}{m_{Z}-2 E_{\ell}} .
$$


The partial width of the $Z$ boson is then given by,

$$
\Gamma\left(Z \rightarrow \bar{\nu}_{\ell} \mathcal{U}\right)=\sum_{\alpha=1}^{\mathcal{N}_{N}} m_{Z} \frac{\left(g^{2}+g^{\prime 2}\right)\left|U_{N_{\alpha} \ell}\right|^{2}}{192 \pi^{2} C_{\lambda}^{2}}\left(\frac{M_{N}}{m_{Z}}\right)^{2}\left(\frac{m_{Z}}{\Lambda}\right)^{2 \Delta_{\mathrm{N}}-3} A_{\Delta_{\mathrm{N}}-1 / 2} f\left(\Delta_{\mathrm{N}}, \frac{\mu_{\mathrm{IR}}^{2}}{m_{Z}^{2}}\right) .
$$

\subsection{Current constraints and future reach from HNL searches}

In this subsection we determine the current bounds on this class of models by recasting existing searches for the decay of $W$ and $Z$ bosons into elementary HNLs that mix with the SM neutrinos. We also discuss the expected reach of similar searches in the future. Our results are obtained under the assumption that all decays into the hidden sector result in the production of just a single composite singlet neutrino, which allows us to compare the rates for unparticle production to the corresponding rates for production of elementary HNLs [52]. Although the resulting limits will be stronger than the actual bounds, the differences are expected to be modest since the results of the previous subsection indicate that decays into states with very few composite singlet neutrino are heavily favored.

In our analysis, we consider only the bounds coming from final states that include electrons, which tend to be the strongest in flavor-democratic models. Furthermore, for simplicity we assume that all the $N_{\alpha}$ are degenerate, $M_{N_{\alpha}}=M_{N}$, and present the bound in terms of the effective mixing angle squared,

$$
\left|U_{N e}\right|^{2} \equiv \sum_{\alpha=1}^{\mathcal{N}_{N}}\left|U_{N_{\alpha} e}\right|^{2}
$$

The current constraints on this class of models are displayed in figure 7 . The constraints are expressed as limits on the mixing angle $U_{N e}$ as a function of the mass $m_{N}$ of the composite singlet neutrino. The bounds are shown for four different choices of the scaling dimension $\Delta_{\mathrm{N}}$. The limits from $W$ and $Z$ boson decay shown in the figure are based on the following searches:

- $L H C$ : above a few $\mathrm{GeV}$ the constraints come from the current LHC searches for promptly decaying HNLs produced in $W$ boson decays [53, 54], shown as the brown curves.

- LEP: LEP dominates the current constraints in the $2-40 \mathrm{GeV}$ regime. The bounds on HNL production from rare $Z$ boson decays searched for by the DELPHI experiment at LEP [55] are shown in blue. The LEP search covers a broad range of signature spaces, including HNLs giving rise to prompt decays, displaced vertices and energy deposition in the calorimeters. In our analysis, we consider only the bounds from prompt decays, since the constraints from long-lived particle searches at LEP are weaker than those from a combination of prompt searches at LEP and D-meson decays at beam dumps.

The future reach of HNL searches for this class of models is shown in figure 8. In the figure the current limits in the composite neutrino mass versus mixing angle plane are 

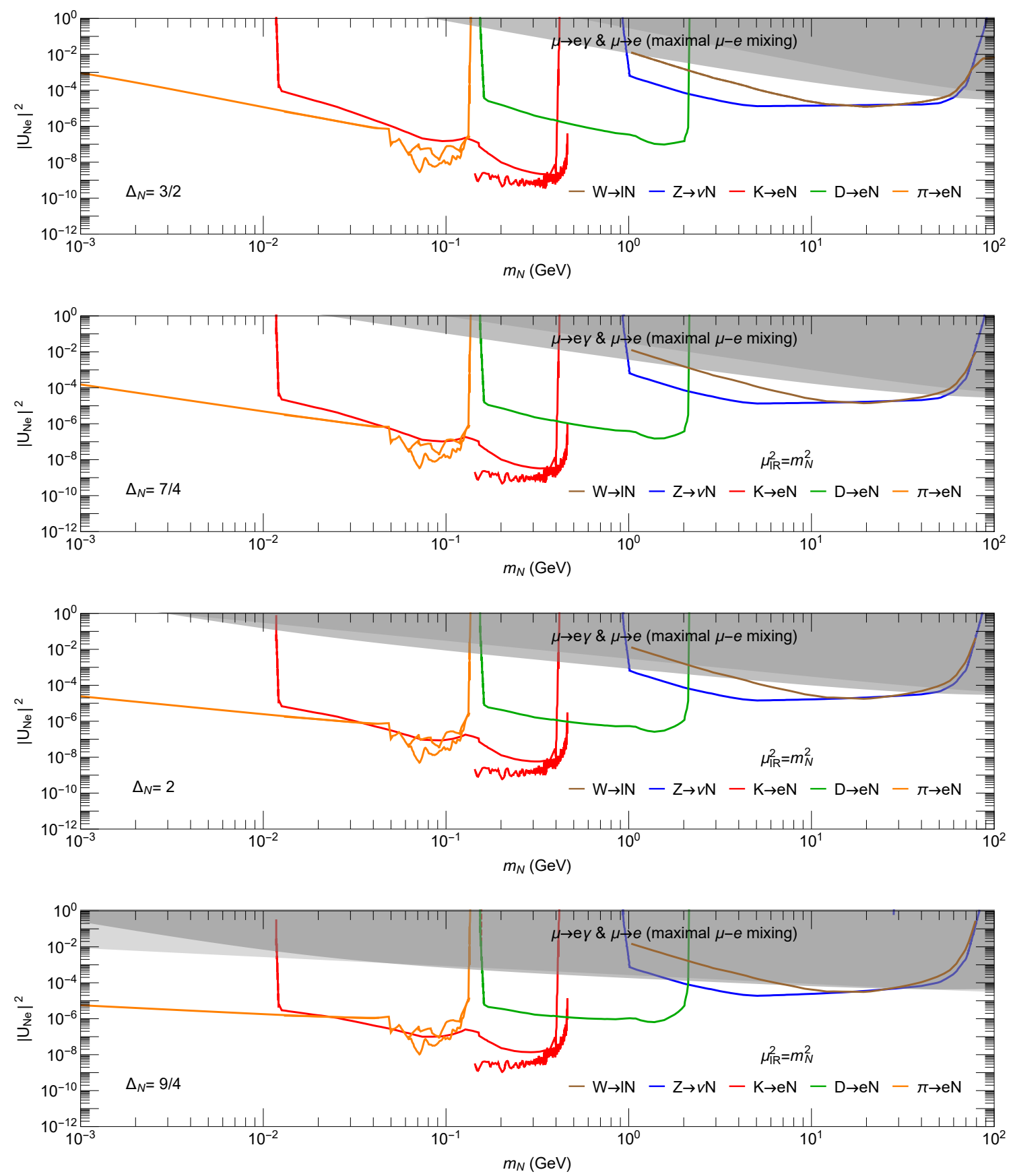

Figure 7. The existing limits on the IR mixing angle as a function of composite neutrino mass $\left(m_{N} \sim \Lambda\right.$, the compositeness scale) for final states involving electrons. These bounds are a reinterpretation of constraints on HNLs, as discussed in the text. The lower and upper shaded gray regions correspond to the flavor dependent constraints from the $\mu \rightarrow e$ conversion and $\mu \rightarrow e \gamma$ measurements in the maximal electron-muon flavor mixing scenario, which is discussed in the next section. 

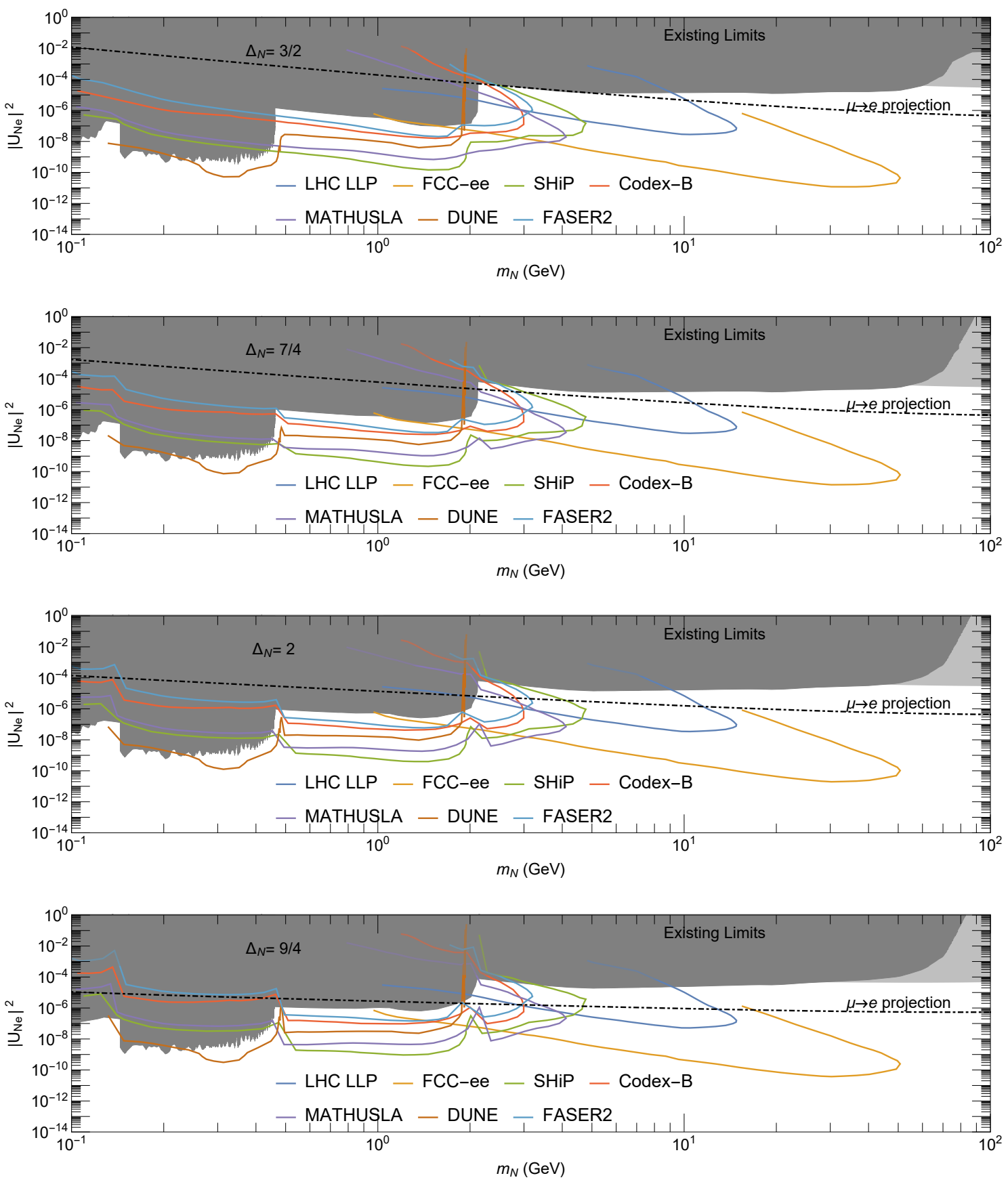

Figure 8. Future projections for constraints on the composite neutrino parameter space, in the singlet neutrino mass $m_{N}$-mixing angle squared $\left|U_{N e}\right|^{2}$ plane, for different benchmark choices of the scaling dimensions $\Delta_{\mathrm{N}}$. The current limits detailed in figure 7 are shaded in gray. Details of the future projections can be found in the text. The flavor dependent current limit and future projections from the $\mu$-e conversion experiments are in light gray shade and dot-dashed line, respectively. 
shaded in gray, with the flavor-dependent constraints in a lighter shade. The projected future limits from $W$ and $Z$ bosons decays shown in the figure are based on the following proposed searches:

- $H L-L H C$ : the projected HL-LHC reach is shown in figure 8 as the blue curves, using a conservative analysis of long-lived particles produced in $W$-boson decays [56]. The analysis employs a dilepton trigger with an additional displaced lepton as the signal, based on the calibration data for the displaced dilepton analysis carried out by CMS [57]. New search ideas at the HL-LHC will be able to further improve the coverage. For a more optimistic projection without background, see, e.g., refs. [58, 59].

- FCC-ee: projections for FCC-ee are shown in orange in figure 8, based on a long-lived particle analysis from its $Z$-pole run [60].

\subsection{Novel signatures}

This class of theories can give rise to exotic signals that are not present in conventional models with an elementary singlet neutrino. If the $W$ boson mass is hierarchically larger than the compositeness scale, $m_{W} \gg \Lambda$, multiple singlet neutrinos can be produced in the same decay, $W^{ \pm} \rightarrow \ell^{ \pm}+n N$, where $n$ is an odd number. These composite singlet neutrinos are not stable, but decay to SM particles through the weak interactions. At colliders, decays to final states that contain charged leptons, $N \rightarrow \nu+\ell^{+}+\ell^{-}$, are especially promising. In certain regions of parameter space the singlet neutrinos are long-lived, leading to striking displaced vertex signatures that have not been constrained in previous experiments [54$56,58,61,62]$. Therefore, in this class of models, the decays of $W$ bosons can give rise to highly characteristic signals involving multiple displaced vertices. Since the LHC is expected to produce of order $10^{12} \mathrm{~W}$ bosons over its lifetime, it is expected to have excellent reach for this class of models in much of parameter space.

The lifetime of the composite singlet neutrinos is given approximately given by,

$$
\begin{aligned}
c \tau_{\alpha} & \approx\left(\sum_{\ell} \kappa_{\ell} \frac{G_{F}^{2} m_{N}^{5}}{192 \pi^{3}}\left|U_{N_{\alpha} \ell}\right|^{2}\right)^{-1} \\
& \sim 60 \times\left(\frac{\mathrm{GeV}}{M_{N}}\right)^{5}\left(\frac{0.01 \mathrm{eV}}{m_{\nu}}\right)\left(\frac{\mu^{c}}{\mathrm{keV}}\right) \text { meter. }
\end{aligned}
$$

The parameter $\kappa_{i}$ counts, for a specific lepton flavor $i$, the number of kinematically allowed decay channels mediated by the off-shell $W$, as well as the number of appropriately weighted decay channels mediated by the off-shell $Z{ }^{1}$ For a composite singlet neutrino mass of $M_{N}=1 \mathrm{GeV}, \kappa_{e} \approx \kappa_{\mu} \approx 8.25$. The $Z$-mediated process also contributes to $\kappa_{\tau} \approx 2.8$. The mixing angle scales as $\left|U_{N_{\alpha} \ell}\right|^{2} \sim m_{\nu} / \mu^{c}$ and corresponds to a benchmark value of $10^{-5}$ for the above choices of $m_{\nu}$ and $\mu^{c}$. In our analysis we use the results presented in ref. [63] to model the details of threshold effects, finite fermion mass corrections, etc., on the lifetime of the composite singlet neutrinos.

\footnotetext{
${ }^{1}$ In calculating $\kappa_{i}$, it is necessary to take into account interference between the $W$ and $Z$-mediated channels when considering final states involving charged leptons.
} 

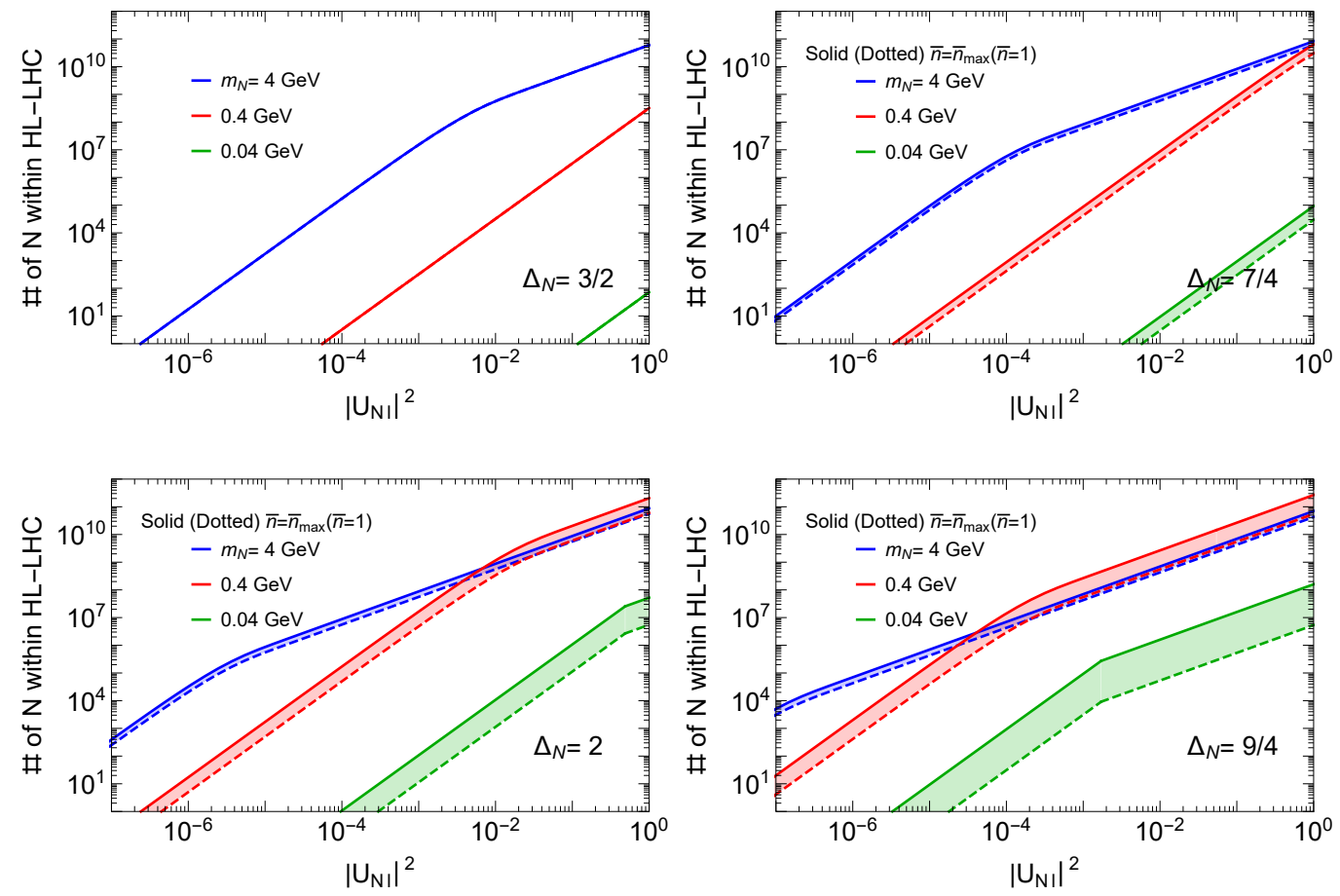

Figure 9. The expected number of composite neutrinos decays at the HL-LHC for various benchmark choices of the $m_{N}$ and scaling dimensions $\Delta_{\mathrm{N}}$. The solid or dashed lines show how the results vary by assuming each unparticle produced results in $n_{\max }$ or one singlet neutrino, respectively.

Given the large proper lifetime of the composite singlet neutrinos and their energy distribution as indicated from figure 6 , we can place an approximate upper limit on the total number of composite singlet neutrino decay events within a detector radius $R_{\text {LHC }}$ at the HL-LHC as,

$$
\left\langle n_{N}\right\rangle \lesssim n_{W} \times \frac{\Gamma_{W}^{\ell N}\left(M_{N}, \Delta_{\mathrm{N}}, \mu_{\mathrm{IR}}\right)}{\Gamma_{W}^{\mathrm{tot}}} \times \bar{n}_{\max }\left(m_{N}, \Delta_{\mathrm{N}}\right) \times\left(1-\exp \left[-\frac{R_{\mathrm{LHC}}}{\gamma\left(\bar{n}_{\max }\right) \beta\left(\bar{n}_{\max }\right) c \tau}\right]\right) .
$$

Here $\bar{n}_{\text {max }}$ represents the maximum number of composite singlet neutrinos that can be produced in the decay of a $W$ boson to the hidden sector after averaging over the kinematics of individual events. In figure 9 we show the total number of composite neutrino decays within a detector radius of $R_{\mathrm{LHC}}=10$ meters at the $13 \mathrm{TeV}$ LHC with an integrated luminosity of $3000 \mathrm{fb}^{-1}$. This figure should be considered together with figure 7 to determine the regions of parameter space that are not already excluded. The results are presented for three different benchmark values of the composite singlet neutrino mass, 0.04, 0.4, and $4 \mathrm{GeV}$, shown in green, red and blue respectively. To explore the dependence of the result on the number of composite singlet neutrinos produced per $W$ decay, we consider two limiting cases. In the first limiting case, this number is taken to be the maximally kinematically allowed value $n_{\max }$ defined in eq. (3.9), averaged over the differential distribution in eq. (3.5). In the second of the two limiting cases, the number of composite singlet neutrinos produced per $W$ decay is simply taken to be 1 . The kinks in the curves correspond to the 
transition between prompt signals (larger mixing angles) and collider metastable signals (smaller mixing angles). We see that in general, if we require about 100 singlet neutrinos decays within the LHC for discovery, the HL-LHC can probe values of the mixing angle $\left|U_{N \ell}\right|^{2}$ in the range from $10^{-7}$ to $10^{-5}$. This shows that the HL-LHC can indeed probe unexplored regions of the parameter space. Note that the phenomenology of unparticle neutrino is closely related to that of dark showers [64-69], where the shower particles are often hidden pions or hidden photons. Instead, our model predicts an HNL shower, and in particular, in lepton(charged and neutral)-rich final states. Here, in addition, the unparticle calculation allows for an approximate prediction of the accompanying charged lepton differential cross section from $W$ decay, which could be an crucial consistency check for the underlying theory and dynamics.

\section{Beam dumps}

For compositeness scales below a few $\mathrm{GeV}$, the rare decays of pions, kaons and B-mesons constitute a powerful probe of this class of models. In this section we discuss the current bounds on this scenario from the rare decays of mesons. In addition, we explore the reach of future beam dump experiments and the proposed LHC-based experiments FASER2, Codex-B and MATHUSLA.

For the meson decay $\mathfrak{m} \rightarrow \ell \mathcal{U}$, the square of the spin averaged matrix element is given by,

$$
|\overline{\mathcal{M}}|^{2}=4 G_{F}^{2}\left|V_{q q^{\prime}}\right|^{2} f_{\mathfrak{m}}^{2}\left(\frac{\hat{\lambda} v_{\mathrm{EW}}}{M_{\mathrm{UV}}^{\Delta_{\mathrm{N}}-3 / 2}}\right)^{2} m_{\mathfrak{m}} E_{\ell},
$$

where $f_{\mathfrak{m}}$ is the meson decay constant. The corresponding partial width is then,

$$
\Gamma(\mathfrak{m} \rightarrow \ell \mathcal{U})=\sum_{\alpha=1}^{\mathcal{N}_{N}} \frac{m_{\mathfrak{m}} G_{F}^{2} f_{\mathfrak{m}}^{2} m_{N}^{2}\left|V_{q q^{\prime}}\right|^{2}\left|U_{N_{\alpha} \ell}\right|^{2}}{32 \pi^{2} C_{\lambda}}\left(\frac{m_{\mathfrak{m}}}{M_{\mathrm{UV}}}\right)^{2 \Delta_{\mathrm{N}}-3} A_{\Delta_{\mathrm{N}}-1 / 2} \times g\left(\Delta_{\mathrm{N}}, \frac{\mu_{\mathrm{IR}}^{2}}{m_{\mathfrak{m}}^{2}}\right),
$$

where $g\left(\Delta_{\mathrm{N}}, \frac{\mu_{\mathrm{IR}}^{2}}{m_{W}^{2}}\right)$ captures the dependence on the scaling dimension $\Delta_{\mathrm{N}}$ and the infrared cutoff $\mu_{\mathrm{IR}}$. The details may be found in the appendix in eq. (A.14). As before, we can express the partial width in terms of the conventional sterile neutrino mixing angle $U_{N \ell}$ using eq. (3.12).

As with the calculation of $W$ and $Z$ boson decays into composite singlet neutrinos detailed in the previous section, we translate the existing constraints on elementary HNLs into constraints on the parameter space of our model. As before, our limits are obtained under the assumption that all decays to the hidden sector result in just a single composite singlet neutrino. This allows the bound to be obtained from a straightforward comparison of the rate for unparticle production to the rate for production of elementary sterile neutrinos [52].

The current bounds on the effective mixing angle squared $\left|U_{N e}\right|^{2} \equiv \sum_{\alpha=1}^{\mathcal{N}_{N}}\left|U_{N_{\alpha} e}\right|^{2}$ as a function of composite neutrino mass $m_{N}$ from rare meson decays are shown in figure 7 . The results are presented for four different choices of $\Delta_{N}$, the scaling dimension. With the exception of the pion decay bound, the charged meson searches look for the decay 
products of elementary HNLs into final states involving charged leptons or charged pions. For concreteness, in our analysis we have only considered constraints arising from final states involving electrons. Since the heavy neutrino provides the chirality flip required for scalar meson decays, and the electron is the lightest charged lepton, these constraints tend to be the strongest in flavor-democratic models. The limits we present are based on the following searches for elementary HNLs.

- TRIUMF: the strongest constraints below the pion mass threshold are from searches at TRIUMF for soft positrons from positively charged pion decays and the overall pion exclusive decay rate measurement [70]. These are shown as the orange curves in figure 7. We also include the bounds from the recent PIENU [71] experiment at TRIUMF shown in the same color which sets a stronger constraint in regions where the limits are provided.

- PS191: searches for charged kaon decays into a charged lepton plus a HNL performed at CERN's PS191 experiment [72] lead the constraints in the $130-400 \mathrm{MeV}$ regime. These are shown in red in figure 7 .

- CHARM: searches for HNLs from D-meson decays were conducted at the CHARM experiment [73]. The resulting bounds are shown in green in figure 7 .

- NA62: the NA62 [74] experiment searched for long-lived HNL decays from a high intensity beam dump with $1.74 \times 10^{18}$ Proton-on-Target (PoT). It is sensitive to HNLs produced in kaon decays, leading to the limits shown as the lower red curves in figure $7 .^{2}$

In figure 8 we show the projected reach of future experiments. In this plot, the existing limits on the parameter space are shaded in gray. Included in the figure are the projected sensitivities to composite singlet neutrinos produced from meson decays in several different future experiments, including SHiP, Codex-B, MATHUSLA, DUNE and FASER-2. Most of the projections are based on ref. [60].

- SHiP: the SHiP experiment [76], which has a higher beam energy than other beam dump proposals, can probe long-lived HNL decays. It is sensitive to HNL masses up to $5 \mathrm{GeV}$ from the decays of B-mesons. The sensitivity is shown as the green curves in figure 8.

- DUNE: the DUNE near detector can search for the decays of long-lived particles produced from the beam dump used to produce neutrinos [77]. The highly intense beam with $3 \times 10^{22}$ PoT can copiously produce $D$-mesons, kaons and pions, all of which can act as sources for singlet neutrinos. DUNE has greater sensitivity reach below the kaon mass threshold than other proposals, as can be seen from figure 8 .

\footnotetext{
${ }^{2}$ T2K ND280 [75] also searched for HNLs from kaon decays. The resulting limits are weaker than those from NA62 and we do not explicitly include them here.
} 

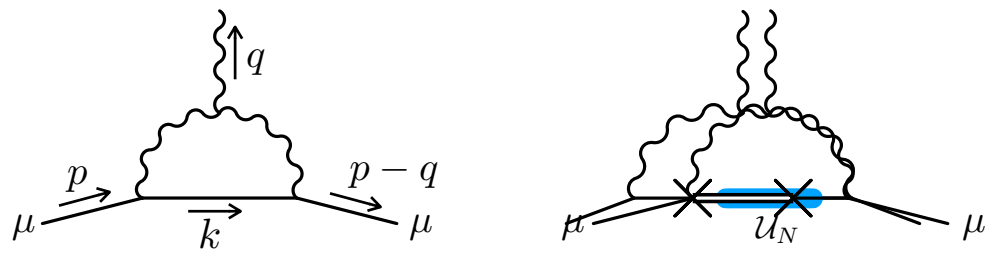

Figure 10. Diagrams contributing to $(g-2)_{\mu}$ with an elementary neutrino in the loop (left), and the correction to this from unparticles (right). Our momentum labels are shown for the elementary case. Similar diagrams with an outgoing electron on the external leg will contribute to $\mu \rightarrow e \gamma$.

- FASER2, Codex-B, MATHUSLA: the projected sensitivities of these LHC satellite experimental proposals are shown in figure 8 as the blue, orange, and purple curves respectively. These limits, which are taken from ref. [60], arise from the decays of mesons into long-lived HNLs.

One complication in translating the limits on elementary singlet neutrinos into bounds on our model arises from the fact that, in general, each of these future experiments presents their results after combining all the different meson production modes. It then becomes a challenge to scale the results appropriately for each of the different contributions. In our analysis we have assumed that production through decays of the lightest available meson dominates, which is true for most of the parameter regions. For instance, in the DUNE projections, in the case of singlet neutrino masses above the kaon mass we assume the limits are entirely from $D$ meson decays, while for singlet neutrino masses between the pion and kaon masses, we take the limits as arising purely from kaon decays (neglecting the contribution from $D$ mesons).

\section{Muon magnetic moment and lepton flavor violation}

Through its couplings to the SM neutrinos the hidden sector contributes at loop level to the anomalous magnetic moment of the muon and to lepton flavor violating processes. In this section we determine the size of these effects and explore the prospects for future experiments to detect them.

The measured value of the anomalous magnetic moment of the muon differs at approximately $\sim 4 \sigma$ from the SM prediction $[78,79]$. This long standing anomaly can be explained by the presence of new light states and will soon be tested by Fermilab's E989 [80]. The lepton flavor violating processes $\mu \rightarrow e \gamma$ and $\mu-e$ conversion will also be probed with increased precision at the upcoming COMET and Mu2e experiments [81, 82]. The calculation of composite neutrino contributions to these processes is quite similar. We focus first on the calculation of $(g-2)_{\mu}$ and on lepton flavor violation in the next subsection.

\subsection{Muon magnetic moment}

A neutral fermion of mass $m_{f}$ that couples to the muon and the $W$ boson gives rise to a one loop contribution to $(g-2)_{\mu}$ through the diagram shown on the left of figure 10 . The 
resulting one loop amplitude can be written in the form,

$$
\bar{u}\left(p-q, m_{\mu}\right) \Gamma^{\alpha}(p-q, p) u\left(p, m_{\mu}\right)
$$

where $q$ represents the momentum of the outgoing photon. In unitary gauge the expression for $\Gamma^{\alpha}(p-q, p)$ is proportional to,

$$
\int \frac{d^{4} k}{16 \pi^{2}} \frac{\gamma^{\nu} P_{L} \not k \gamma^{\mu} P_{L}}{\left(k^{2}-m_{f}^{2}\right)} D_{F \mu \lambda}(k-p) D_{F \delta \nu}(k-p+q) V^{\lambda \delta \alpha}(k-p, k-p+q, q)
$$

where $D_{F \mu \lambda}(k-p)$ and $D_{F \delta \nu}(k-p+q)$ represent the $W$ boson propagators and $V^{\lambda \delta \alpha}(k-$ $p, k-p+q, q)$ denotes the contribution to the amplitude from the triple gauge boson vertex. We can decompose $\Gamma^{\alpha}(p-q, p)$ as,

$$
\Gamma^{\alpha}(p-q, p)=\gamma^{\alpha} F_{1}\left(q^{2}\right)+\frac{i \sigma^{\alpha \beta} q_{\beta}}{2 m_{\mu}} F_{2}\left(q^{2}\right)
$$

where $F_{1}\left(q^{2}\right)$ and $F_{2}\left(q^{2}\right)$ are form factors. In this expression the anomalous magnetic moment arises from the contribution proportional to $\sigma^{\alpha \beta} q_{\beta}$. This effect has been calculated [83],

$$
a_{\mu}=\frac{G_{F} m_{\mu}^{2}}{8 \sqrt{2} \pi^{2}} \int_{0}^{1} d x \frac{2 x\left(2 r_{f}^{2}+x^{2}\left(r_{f}^{2}+r_{\mu}^{2}+2\right)-x\left(3 r_{f}^{2}+r_{\mu}^{2}-2\right)\right)}{r_{\mu}^{2} x^{2}+r_{f}^{2}-x\left(r_{f}^{2}+r_{\mu}^{2}-1\right)}
$$

where $r_{f, \mu} \equiv m_{f, \mu} / M_{W}$. We denote the integral over $x$ as $I\left(r_{f}, r_{\mu}\right)$. Expanding in the small parameter $r_{\mu}$ leads to a tractable integral with

$$
I\left(r_{f}, r_{\mu}\right)=\frac{10-43 r_{f}^{2}+78 r_{f}^{4}-49 r_{f}^{6}+4 r_{f}^{8}+18 r_{f}^{6} \log r_{f}^{2}}{3\left(r_{f}^{2}-1\right)^{4}}+\mathcal{O}\left(r_{\mu}^{2}\right) .
$$

We now turn our attention to the case with unparticles in the loop. If we work in the insertion approximation, the contribution to the muon magnetic moment arises from a diagram of the form shown on the right of figure 10 that contains two insertions of the neutrino-composite neutrino mixing operator given in eq. (2.3). The single fermion propagator in (5.2) is now replaced by two massless neutrino propagators and an unparticle propagator. For the range of scaling dimensions of interest, $3 / 2 \leq \Delta_{\mathrm{N}}<5 / 2$, we can express the unparticle propagator as an integral over a spectral function,

$$
\Delta(p)=\frac{A_{\Delta_{\mathrm{N}}-1 / 2}}{2 \pi} \int_{\mu_{\mathrm{IR}}^{2}}^{\infty} d M^{2}\left(M^{2}-\mu_{\mathrm{IR}}^{2}\right)^{\Delta_{\mathrm{N}}-5 / 2} \frac{i \not p}{p^{2}-M^{2}+i \epsilon},
$$

with $A_{n}$ as given in eq. (2.5). Since the conformal symmetry is broken at low scales it is necessary to cut off this integral. We have chosen to do this in a simple way following [84], cutting off contributions to the spectral function in the infrared from scales below $\mu_{\mathrm{IR}}^{2} \sim \Lambda^{2}$. Then the contribution of the unparticle sector to the muon magnetic moment can be obtained by making the replacement,

$$
\frac{i \not k}{k^{2}-m_{f}^{2}} \longrightarrow\left(\frac{\lambda v}{C_{\lambda} \Lambda^{\Delta_{N}-3 / 2}}\right)^{2} \frac{A_{\Delta_{N}-1 / 2}}{2 \pi} \int_{\mu_{\mathrm{IR}}^{2}}^{\infty} d M^{2}\left(M^{2}-\mu_{\mathrm{IR}}^{2}\right)^{\Delta_{\mathrm{N}}-5 / 2} \frac{-i \not k k k}{k^{2}\left(k^{2}-M^{2}\right) k^{2}},
$$


in eq. (5.2). The insertion of the mixing angles and the additional propagators makes the loop integral more complicated. However, taking advantage of the identities $k k k=k^{2}$ and

$$
\frac{1}{k^{2}\left(k^{2}-M^{2}\right)}=\frac{1}{M^{2}}\left(\frac{1}{k^{2}-M^{2}}-\frac{1}{k^{2}}\right)
$$

the unparticle diagram can be evaluated as an integral over the difference of two simpler diagrams,

$$
a_{\mu}^{\mathcal{U}}=\frac{G_{F} m_{\mu}^{2}}{16 \sqrt{2} \pi^{2}}\left(\frac{\lambda v}{C_{\lambda} \Lambda^{\Delta_{\mathrm{N}}-3 / 2}}\right)^{2} \frac{A_{\Delta_{\mathrm{N}}-1 / 2}}{2 \pi} \int_{\mu_{\mathrm{IR}}^{2}}^{\infty} \frac{d M^{2}}{M^{2}}\left(M^{2}-\mu_{\mathrm{IR}}^{2}\right)^{\Delta_{\mathrm{N}}-5 / 2} \Delta I,
$$

where $\Delta I \equiv I\left(M / M_{W}, m_{\mu} / M_{W}\right)-I\left(0, m_{\mu} / M_{W}\right)$. Again, expanding in the small parameter $m_{\mu} / M_{W}$ leads to a tractable result

$$
\begin{aligned}
a_{\mu}^{\mathcal{U}}= & -\sum_{\alpha=1}^{\mathcal{N}_{N}} \frac{G_{F} m_{\mu}^{2}}{16 \sqrt{2} \pi^{3}}\left(\frac{\left|U_{N_{\alpha}}\right|^{2}}{C_{\lambda}^{2}}\right)\left(\frac{M_{W}}{M_{N}}\right)^{2 \Delta_{\mathrm{N}}-5} A_{\Delta_{\mathrm{N}}-1 / 2} \\
& \times \int_{z_{\mathrm{IR}}}^{\infty} d z\left(z^{2}-z_{\mathrm{IR}}^{2}\right)^{\Delta_{\mathrm{N}}-5 / 2}\left[\frac{2 z^{7}+3 z^{5}-6 z^{3}+z-6 z^{5} \log \left(z^{2}\right)}{\left(z^{2}-1\right)^{4}}\right],
\end{aligned}
$$

with $z_{\mathrm{IR}}=\mu_{\mathrm{IR}} / M_{W}$ and $\Lambda \sim m_{N}$. Unfortunately, this is of the wrong sign to explain the discrepancy in the muon anomalous magnetic moment [85]. It therefore places a constraint on the parameter space of the model. The observed excess in the muon anomalous magnetic moment is $\Delta a_{\mu}=(31.3 \pm 7.7) \times 10^{-10}$. Using the CLs method and requiring that the composite neutrino model hypothesis not be excluded at the $95 \%$ confidence level as compared to the SM-only hypothesis, we are able to place a bound on the hidden sector contribution, $\left|a_{\mu}^{\mathcal{U}}\right| \lesssim 5 \times 10^{-10}$. This translates into limits on the mixing angle $\left|U_{N \mu}\right|$ shown as the red lines in figure 11 .

\subsection{Lepton flavor violation}

In general, the couplings of the hidden sector to the SM neutrinos are not expected to be flavor aligned and can therefore give rise to flavor violating processes involving the charged leptons. The lepton flavor violating muon decay $\mu \rightarrow e \gamma$ and the $\mu-e$ conversion process will be probed with greatly increased precision at the upcoming COMET and Mu2e experiments $[81,82]$. In this subsection we determine the hidden sector contributions to these processes.

The process $\mu \rightarrow e \gamma$ arises from a diagrams of the same form as in figure 10. The resulting amplitude can be evaluated in the exactly same way, resulting in a contribution to the branching ratio given by,

$$
\begin{aligned}
B R^{\mathcal{U}}(\mu \rightarrow e \gamma)= & \left(\frac{3 \alpha}{128 \pi^{3} C_{\lambda}^{4}}\right) \frac{\left|\sum_{\alpha=1}^{\mathcal{N}_{N}} U_{N_{\alpha} \mu}^{*} U_{N_{\alpha} e}\right|^{2}}{M_{N}^{4 \Delta_{N}-6}} \\
& \times\left(A_{\Delta_{\mathrm{N}}-\frac{1}{2}} \int_{\mu_{\mathrm{IR}}^{2}}^{\infty} \frac{d M^{2}}{M^{2}}\left(M^{2}-\mu_{\mathrm{IR}}^{2}\right)^{\Delta_{\mathrm{N}}-5 / 2} \Delta I\right)^{2} .
\end{aligned}
$$




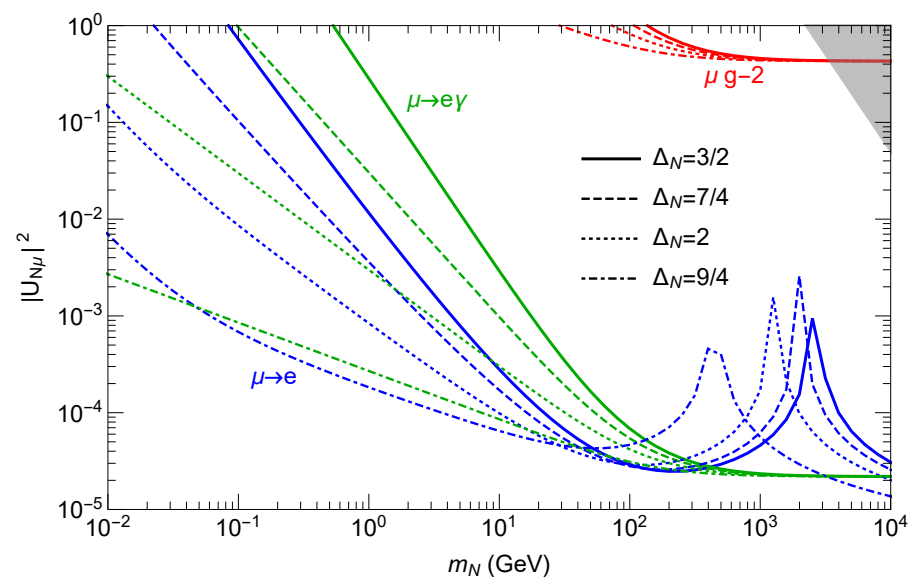

Figure 11. Constraints on neutrino mixing as a function of the singlet neutrino mass scale from muon g-2 (red lines), $\mu \rightarrow e \gamma$ (green lines), and $\mu$-e conversion (blue lines) experimental measurements, for benchmark choices of $\Delta_{\mathrm{N}}$ of $3 / 2$ (solid lines), $7 / 4$ (dashed lines), 2 (dotted lines), 9/4 (dot-dashed lines). In the gray region the boundary condition in eq. (2.18) is violated.
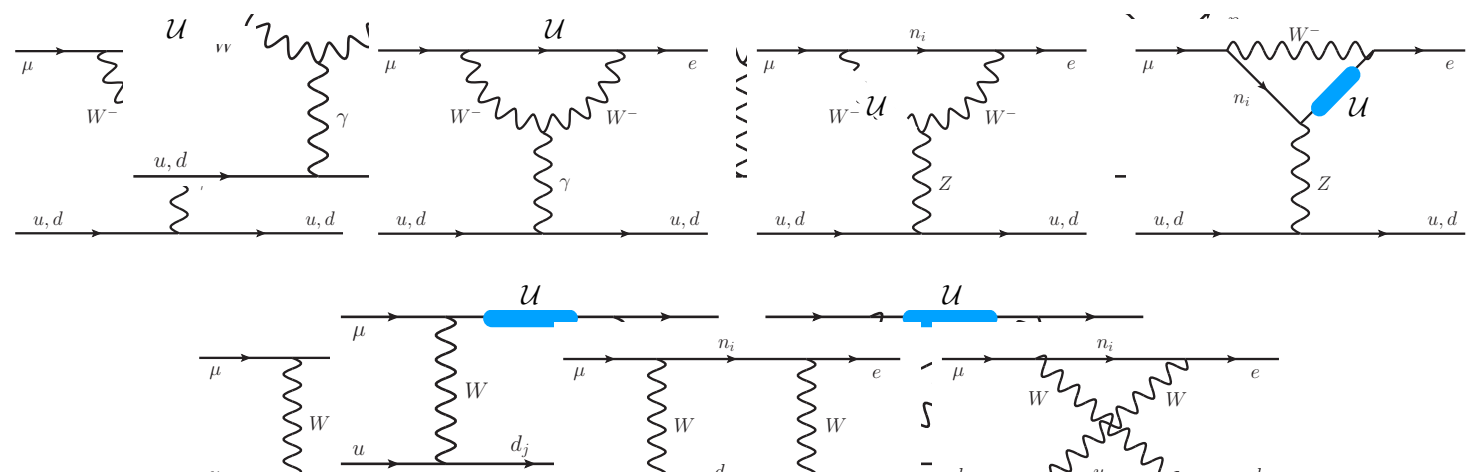

Figure 12. Loop diagrams contributing to $\mu \rightarrow e$ transitions with unparticle neutrino particles in the loop.

The current limit on this branching ratio from the MEG experiment [86] places a constraint on the matrix product $\left|\sum_{\alpha=1}^{\mathcal{N}_{N}} U_{N_{\alpha} \mu}^{*} U_{N_{\alpha}} e\right|^{2}$. This is shown in figure 7 as a bound on $\mid U_{N}$ e $\mid$ alone, under the simplifying assumption $\left|\sum_{\alpha=1}^{\mathcal{N}_{N}} U_{N_{\alpha} \mu}^{*} U_{N_{\alpha}}\right|^{2}=\left|U_{N e}\right|^{2}$.

There are several one loop diagrams that contribute to $\mu-e$ conversion. They are shown in figure 12. They include $W$-box, penguin and $Z$-penguin diagrams. As before, we work in the insertion approximation. In this limit the amplitudes of the individual diagrams can be related to the corresponding amplitudes for the case of elementary HNLs propagating in the loop. This rate, $R_{\mu \rightarrow e}$, has been carefully calculated in [87] and is given by,

$$
R_{\mu \rightarrow e}=\frac{2 G_{F}^{2} \alpha_{W}^{2}}{\pi^{2} \Gamma_{\text {capt }}}\left(\frac{V^{(p)}}{Z}\right)^{2}\left|(A+Z) F_{u}+(2 A-Z) F_{d}\right|^{2},
$$

for a nucleus of atomic number $Z$, mass number $A$ and muon capture rate $\Gamma_{\text {capt }}$. The 
details of the nuclear form factor are encoded in $V^{(p)}$. The functions $F_{u, d}$, given in [87], are form factors determined by the one loop diagrams and are a function of the mass of the HNL. There is a value of the neutrino mass for which the neutron and proton contributions approximately cancel, $F_{u} / F_{d}=(Z-2 A) /(A+Z) \approx-1$, and the rate for muon conversion is suppressed, resulting in a drop in sensitivity for that element at that mass. As before, for the case of unparticles running in the loop we make the replacement,

$$
\begin{aligned}
F_{u, d} \rightarrow & \sum_{\alpha=1}^{\mathcal{N}_{N}} U_{N_{\alpha} \mu}^{*} U_{N_{\alpha} e}\left(\frac{M_{N}}{\sqrt{2} M_{W} C_{\lambda}}\right)^{2}\left(\frac{M_{W}}{\Lambda}\right)^{2 \Delta_{\mathrm{N}}-3} \\
& \times \frac{A_{\Delta_{\mathrm{N}}-\frac{1}{2}}}{2 \pi} \int_{x_{\mathrm{IR}}}^{\infty} d x \frac{\left(x-x_{\mathrm{IR}}\right)^{\Delta_{\mathrm{N}}-5 / 2}}{x} F_{u, d}(x)
\end{aligned}
$$

with $x_{\mathrm{IR}}=\left(\mu_{\mathrm{IR}} / M_{W}\right)^{2}$. Cancellations between the neutron and proton contributions to the rate can still occur in the case of composite neutrinos, but at masses that are shifted relative to the case of elementary singlet neutrinos.

Currently, the strongest bound on $R_{\mu \rightarrow e}$ comes from the SINDRUM II experiment [88], which is based on measurements on gold. This limit stands at $R_{\mu \rightarrow e}^{A u}<7 \times 10^{-13}$ (90\% CL) but is expected to be considerably improved by COMET [81] and Mu2e [82, 89] to the level of $R_{\mu \rightarrow e}^{A l} \lesssim 10^{-16}$. In figure 11 we show the current constraints from $\mu \rightarrow e \gamma$ and $\mu-e$ conversion in green and blue lines, respectively, over a broad range of composite singlet neutrino masses $M_{N}$. These bounds are prsented as limits on $\left|U_{N} \mu\right|$, under the simplifying assumption $\left|\sum_{\alpha=1}^{\mathcal{N}_{N}} U_{N_{\alpha} \mu}^{*} U_{N_{\alpha} e}\right|^{2}=\left|U_{N \mu}\right|^{2}$. We can see from the figure the complementarity between these two types of lepton flavor violating experiments.

In figures 7 and 8 we present the current and future constraints on this class of models, with the limits from the lepton flavor violating processes $\mu \rightarrow e \gamma$ and $\mu-e$ conversion included. The bounds are presented as limits on $U_{N e}$, under the assumption $\left|\sum_{\alpha=1}^{\mathcal{N}_{N}} U_{N_{\alpha} \mu}^{*} U_{N_{\alpha}} e\right|^{2}=\left|U_{N e}\right|^{2}$. We see that in the limit of large mixing, $\mu-e$ conversion currently gives the strongest bound on this class of models for $M_{N} \gtrsim 70 \mathrm{GeV}$. In the future, assuming large mixing, Mu2e will provide greater sensitivity than the current limits for compositeness scales above a few $\mathrm{GeV}$ and will have the greatest reach of all future experiments above $50 \mathrm{GeV}$. Future collider and beam dump experiments are expected to have greater sensitivity for $M_{N}$ below $50 \mathrm{GeV}$, showing the complementarity of these different types of searches. In the event of a discovery, multiple measurements from different experiments may help uncover the nature of the underlying theory.

\section{Neutrinoless double beta decay}

In this section we consider the implications of this class of models for neutrinoless double beta decay. At scales below the compositeness scale $\Lambda$, the spectrum of light states in the neutrino sector consists of just the SM neutrinos and antineutrinos, which now possess small Majorana masses from the nonrenormalizable Weinberg operator. The characteristic momentum scale in neutrinoless double beta decay is of order $100 \mathrm{MeV}$, which corresponds to the spacing between nucleons in the nucleus. Since the Weinberg operator is the lowest 
dimension term that can be added to the SM which violates lepton number, it follows that if the compositeness scale $\Lambda$ lies well above $100 \mathrm{MeV}$, the rate for neutrinoless double beta decay is determined by the mass matrix of the light neutrinos in the low energy effective theory, just as in conventional high scale seesaw models. However, if $\Lambda$ lies below a $100 \mathrm{MeV}$, the situation is more complicated, and the effects of the strong dynamics must be taken into account when calculating the rate for neutrinoless double beta decay.

Before determining the rate for neutrinoless double beta decay in this scenario, it is useful to first consider this process in the context of inverse seesaw models. This has been studied in, for example, [90, 91]. In this framework the rate for neutrinoless double beta decay depends on the lepton number violating contribution to the off-shell neutrino propagator. As can be seen from the Lagrangian for the inverse seesaw, eq. (2.15), this depends on a matrix element of the form,

$$
\mathcal{M}_{N}=\left\langle\nu(p)\left|T\left[\lambda L(x) H(x) N(x) \frac{\mu^{c}}{2}\left(N^{c}(y)\right)^{2} \lambda L(z) H(z) N(z)\right]\right| \nu(p)\right\rangle,
$$

where we are treating the lepton number violating parameter $\mu^{c}$ in the insertion approximation. Setting the Higgs to its VEV, we see that for $M_{N} \gg p$ this matrix element scales as

$$
\mathcal{M}_{N} \sim \frac{\lambda^{2} v_{\mathrm{EW}}^{2} \mu^{c}}{M_{N}^{2}}=m_{\nu}
$$

Therefore, for $M_{N}$ much greater than $100 \mathrm{MeV}$, the rate for neutrinoless double beta decay in inverse seesaw models is set by the masses of the light neutrinos, exactly as expected from effective field theory considerations.

If a term of the form shown in eq. (2.19) is added to the inverse seesaw model, there is an additional contribution to neutrinoless double beta decay proportional to the lepton number violating parameter $\mu$. The corresponding matrix element takes the form,

$$
\widetilde{\mathcal{M}}_{N}=\left\langle\nu(p)\left|T\left[\lambda L(x) H(x) N(x) \frac{\mu}{2} N^{\dagger}(y) \lambda L(z) H(z) N(z)\right]\right| \nu(p)\right\rangle,
$$

where, as before, we are treating $\mu$ in the insertion approximation. Setting the Higgs to its vacuum expectation value (VEV) we see that for $M_{N} \gg p$ this matrix element scales as

$$
\widetilde{\mathcal{M}}_{N} \sim \frac{\lambda^{2} v_{\mathrm{EW}}^{2} \mu}{M_{N}^{2}} \frac{p^{2}}{M_{N}^{2}}=\frac{\mu}{\mu^{c}} \frac{p^{2}}{M_{N}^{2}} m_{\nu}
$$

For $\mu \sim \mu^{c}$ and $M_{N} \gg p$, we see that this contribution to neutrinoless double beta decay is suppressed compared to that in eq. (6.2). This is exactly as expected from effective field theory considerations, since the contribution in eq. (6.4) must necessarily arise from an operator of higher dimension than the Weinberg operator.

We now turn our attention to the opposite limit, $M_{N} \ll p$. The matrix element in eq. (6.1) now scales as,

$$
\mathcal{M}_{N} \sim \frac{\lambda^{2} v_{\mathrm{EW}}^{2} M_{N}^{2} \mu^{c}}{p^{4}}=m_{\nu}\left(\frac{M_{N}}{p}\right)^{4} .
$$


Comparing this to the matrix element in eq. (6.2), it follows that in inverse seesaw models, if the mass of the singlet neutrinos lies below $100 \mathrm{MeV}$, the rate for neutrinoless double beta decay is suppressed. If a term of the form shown in eq. (6.3) is also present, the matrix element in eq. (6.3), in the $m_{N} \ll p$ limit, provides an additional contribution that scales as,

$$
\widetilde{\mathcal{M}}_{N} \sim \frac{\lambda^{2} v_{\mathrm{EW}}^{2} \mu}{p^{2}}=m_{\nu} \frac{\mu}{\mu^{c}}\left(\frac{M_{N}}{p}\right)^{2} .
$$

For $\mu \sim \mu^{c}$ and $M_{N} \ll p$, we see that this contribution to neutrinoless double beta decay, although also suppressed, is larger than that in eq. (6.5). This example serves to illustrate that if the scale at which the neutrino masses are generated lies at or below $100 \mathrm{MeV}$, the rate of neutrinoless double beta decay need not be controlled by the Weinberg operator.

We now consider the scenario in which the singlet neutrinos are composite. Both the Majorana masses of the light neutrinos and the rate for neutrinoless double beta decay arise from the lepton number violating contributions to the neutrino propagator from the composite sector. These are controlled by the matrix element,

$$
\mathcal{M}_{\mathcal{U}}=\left\langle\nu(p)\left|T\left[\hat{\lambda} L(x) H(x) \mathcal{O}_{N}(x) \hat{\mu}^{c} \mathcal{O}_{2 N^{c}}(y) \hat{\lambda} L(z) H(z) \mathcal{O}_{N}(z)\right]\right| \nu(p)\right\rangle .
$$

If the compositeness scale $\Lambda$ lies well above $100 \mathrm{MeV}$, both the neutrino masses and neutrinoless double beta decay depend on the value of this matrix element evaluated at momenta $p \ll \Lambda$. At energies below the compositeness scale $\Lambda$, the matrix element in eq. (6.7) will generate nonrenormalizable lepton number violating interactions among the SM fields. Since the Weinberg operator is the unique lowest dimension lepton number violating operator, effective field theory dictates that the rate for neutrinoless double beta decay will be determined by the form of the mass matrix for the light neutrinos.

If the scale $\Lambda$ lies below $100 \mathrm{MeV}$, the situation is more complicated. While the neutrino masses still depend on the value of the matrix element in eq. (6.7) at scales $p \ll \Lambda$, the rate for neutrinoless double beta decay is now controlled by scales $p \gg \Lambda$. We can estimate the rate for neutrinoless double beta decay by evaluating the matrix element in eq. (6.7) using the general expression for the fermion-fermion-scalar 3-point function in a CFT [92, 93],

$$
\left\langle\mathcal{O}_{\mathrm{N}}\left(x_{1}\right) \mathcal{O}_{\mathrm{N}}\left(x_{2}\right) \mathcal{O}_{2 \mathrm{~N}^{c}}\left(x_{3}\right)\right\rangle=\{\mathbf{I}+\mathbf{I I}\}
$$

where

$$
\mathbf{I}=C_{1} \frac{\left(\not x_{1}-\not{ }_{2}\right)}{\left(x_{13}^{2}\right)^{\Delta_{2 \mathrm{~N}^{\mathrm{c}} / 2}}\left(x_{23}^{2}\right)^{\Delta_{2 \mathrm{~N}^{\mathrm{N}}} / 2}\left(x_{12}^{2}\right)^{\left(2 \Delta_{\mathrm{N}}-\Delta_{2 \mathrm{~N}^{\mathrm{c}}}+1\right) / 2}}
$$

and

$$
\mathbf{I I}=C_{2} \frac{\left(\not x_{1}-\not x_{3}\right)\left(\not \not_{3}-\not x_{2}\right)}{\left(x_{13}^{2}\right)^{\left(\Delta_{2 \mathrm{~N}^{\mathrm{c}}}+1\right) / 2}\left(x_{23}^{2}\right)^{\left(\Delta_{\left.2 \mathrm{~N}^{\mathrm{c}}+1\right) / 2}\left(x_{12}^{2}\right)^{\left(2 \Delta_{\mathrm{N}}-\Delta_{\left.2 \mathrm{~N}^{\mathrm{c}}\right) / 2}\right.}\right.}}
$$

Here $C_{1}$ and $C_{2}$ are undetermined coefficients that depend on the details of the CFT and $\not x \equiv \bar{\sigma}^{\mu} x_{\mu}$. Based on this, we see that the corrections to the neutrino propagator from eq. (6.7) are either ultraviolet divergent or infrared divergent, depending on the scaling dimensions of $\mathcal{O}_{\mathrm{N}}$ and $\mathcal{O}_{2 \mathrm{~N}^{\mathrm{c}}}$. However, if the contributions to the neutrino mass are to be finite in the ultraviolet, we require $2 \Delta_{\mathrm{N}}+\Delta_{2 N^{c}} \leq 8$. Then, using the fact that the neutrino 
mass is generated at scales of order $\Lambda$ to estimate the prefactor, we find that the matrix element in eq. (6.7) scales as

$$
\mathcal{M}_{\mathcal{U}} \sim m_{\nu}\left(\frac{\Lambda^{2}}{p^{2}}\right)^{4-\Delta_{\mathrm{N}}-\Delta_{2 \mathrm{~N}^{\mathrm{C}}} / 2}
$$

Since $2 \Delta_{\mathrm{N}}+\Delta_{2 \mathrm{Nc}^{\mathrm{c}}} \leq 8$, we see that the contribution to neutrinoless double beta decay from scales above $\Lambda$ are suppressed.

It follows from this discussion that for $\Lambda \gg 100 \mathrm{MeV}$, the rate for neutrinoless double beta decay is determined by the neutrino mass matrix, just as in conventional high scale seesaw models. However, for $\Lambda \lesssim 100 \mathrm{MeV}$, the rate for neutrinoless double beta decay is suppressed by form factors, and may be below the reach of next generation experiments.

\section{Cosmology}

In this section we explore the cosmological history of this class of models. We determine the limits that cosmology places on the parameter space of these theories and show that this scenario can lead to observable signals in the CMB.

In the early universe the hidden sector is in thermal contact with the SM, which populates the states in the hidden sector. We find that for $\Lambda \lesssim 1 \mathrm{GeV}$, the couplings between the SM and the hidden sector are large enough that the two sectors are necessarily in thermal equilibrium at the compositeness scale. At temperatures below the compositeness scale, the composite singlet neutrinos annihilate away efficiently into light neutrinos, leaving behind only an exponentially suppressed relic population. These relic singlet neutrinos eventually decay into final states that may include charged leptons. For compositeness scales below about $50 \mathrm{MeV}$, we find that these decays may occur late enough that they happen after the photons in the SM thermal bath have gone out of chemical equilibrium. This results in distortions to the CMB that may be large enough to be observed in future experiments.

Cosmological observations can be used to place constraints on the compositeness scale $\Lambda$. Precision measurements of the CMB have established that the neutrinos are free streaming at temperatures of order an $\mathrm{eV}$ [94-96]. For values of $\Lambda$ less than $100 \mathrm{eV}$, the partially composite character of the neutrinos leads to neutrino self-interactions that are too large to admit free streaming. Therefore these observations disfavor compositeness scales $\Lambda$ below $100 \mathrm{eV}$. In addition, the CMB also places tight limits on the total energy density in radiation during the epoch of acoustic oscillations [97] that can be translated into a lower bound on the compositeness scale. These bounds are usually expressed as limits on the effective number of neutrinos, $N_{\text {eff }}$. We find that the CMB bounds on $N_{\text {eff }}$ disfavor scenarios in which the hidden sector is in equilibrium with the neutrinos at temperatures below an $\mathrm{MeV}$, the scale at which the neutrinos decouple from the rest of the SM. Since the neutrinos are in equilibrium with the hidden sector at temperatures of order the compositeness scale for any $\Lambda \lesssim 1 \mathrm{GeV}$, values of $\Lambda$ below an $\mathrm{MeV}$ are disfavored.

\subsection{Cosmological history}

In the early universe there are several processes that can serve to populate the hidden sector. These include $\nu \nu \leftrightarrow \mathcal{U}, \nu \mathcal{U} \leftrightarrow \mathcal{U}$ and $\nu \mathcal{U} \leftrightarrow \nu \mathcal{U}$. We shall focus on the process $\nu \nu \rightarrow \mathcal{U}$, which does not require an initial population of unparticles. 
We can estimate the inclusive rate for $\nu \nu \rightarrow \mathcal{U}$ by noting that, at momentum scales of order the compositeness scale $\Lambda$, the cross section for this process is expected to be of roughly the same size as the cross section for $\nu \nu \rightarrow N N$. This allows us to estimate the cross section for the process $\nu \nu \rightarrow \mathcal{U}$ at temperatures $T$ of order $\Lambda$ as

$$
\sigma_{\nu \nu \rightarrow \mathcal{U}} \sim \sigma_{\nu \nu \rightarrow N N} \sim \frac{1}{4 \pi}\left(\frac{\kappa}{\Lambda^{2}}\right)^{2}\left(\frac{\lambda v_{\mathrm{EW}}}{M_{N}}\right)^{4} T^{2} .
$$

Here the parameter $\kappa \sim 16 \pi^{2}$. Since the initial state only involves SM neutrinos, which are ultrarelativistic, the rate for this process can be estimated as,

$$
n_{\nu}\left\langle\sigma_{\nu \nu \rightarrow \mathcal{U}} v\right\rangle \sim \frac{\kappa^{2}}{4 \pi}\left(\frac{\lambda v_{\mathrm{EW}}}{M_{N}}\right)^{4} \frac{T^{5}}{\Lambda^{4}}
$$

This process will ensure that the hidden sector is in equilibrium with the SM neutrinos at temperatures of order the compositeness scale provided the rate is faster than the Hubble expansion rate, $H \sim T^{2} / M_{\mathrm{Pl}}$, when $T \sim \Lambda$. Setting $\lambda$ to its lower bound in eq. (2.18), taking $m_{\nu} \sim 0.01 \mathrm{eV}$ and recalling that $M_{N} \sim \Lambda$, we see that this process will necessarily bring the two sectors into equilibrium provided $\Lambda \lesssim 1 \mathrm{GeV}$. For values of $\Lambda \gtrsim 1 \mathrm{GeV}$ this process by itself may not be sufficient to bring the two sectors in equilibrium below the confinement scale. Nevertheless, it will partially populate the CFT states. However, for these higher values of $\Lambda$ the composite singlet neutrinos decay prior to neutrino decoupling, as can be seen from eq. (3.13), and therefore do not leave any observable cosmological effects.

We expect that the hidden sector transitions to the hadronic phase at a temperature $T$ of order the compositeness scale $\Lambda$. Once the temperature falls below the compositeness scale, the hadrons in the hidden sector begin to go out of the bath. The decay of the composite singlet neutrinos is relatively slow, and so the reduction in their number density occurs primarily through annihilation to SM particles. When the strongly coupled sector is in the hadronic phase, the dominant interactions between the SM and hidden sector arise from the mixing between the SM and composite neutrinos in eq. (2.6), when combined with the self-interactions of the $N$, eq. (2.20). As emphasized earlier, the large self-interactions between the singlet neutrinos are characteristic of this framework and distinguish it from conventional inverse seesaw models. These give rise to not just the conventional annihilation process $N N \rightarrow \nu \nu$, but also semi-annihilation $N N \rightarrow N \nu$, and co-annihilation $\nu N \rightarrow \nu \nu$. Since the mixing between the $\mathrm{SM}$ and composite neutrinos is small, $\mathcal{O}\left(\lambda v_{\mathrm{EW}} / M_{N}\right)$, while the dimension-6 self-couplings, which are of order $\mathcal{O}\left(16 \pi^{2} / \Lambda^{2}\right)$, are sizable at temperatures $T$ of order $\Lambda$, the cross sections for these processes diminish with the number of external $\nu$ legs. The conventional annihilation process begins from the same initial state as semi-annihilation, but involves an additional $\nu$ in the final state. It is therefore mixing-suppressed and subdominant relative to semi-annihilation. The coannihilation process, although suppressed by even more powers of $\nu-N$ mixing, can take advantage of the background bath of relativistic SM neutrinos. However, for $\Lambda \gtrsim 1 \mathrm{MeV}$ and mixings allowed by existing constraints (see figure 7), this is not sufficient to overcome the suppression from mixing. 
We therefore focus on the depletion of composite singlet neutrinos through the semiannihilation process, $N N \rightarrow N \nu$. We can obtain an estimate of the relic density of composite singlet neutrinos, which we denote by $n_{F}$, by equating the annihilation rate at freeze out to the Hubble expansion rate,

$$
\left.n_{F}\left\langle\sigma_{N N \rightarrow \nu N} v\right\rangle\right|_{T_{F}}=\left.H\right|_{T_{F}} .
$$

Here $T_{F}$ denotes the temperature at freeze out. We estimate the annihilation rate as,

$$
\left.\left\langle\sigma_{N N \rightarrow N \nu} v\right\rangle\right|_{T_{F}} \sim \frac{\kappa^{2}}{4 \pi} \frac{\lambda^{2} v_{\mathrm{EW}}^{2}}{\Lambda^{4}} \gtrsim \frac{\kappa^{2}}{4 \pi} \frac{m_{\nu}}{M_{N}^{3}} .
$$

To obtain the inequality we have set $\lambda$ to its lower bound in eq. (2.18) and used the fact that $M_{N} \sim \Lambda$. The non-relativistic number density of $N$ at freeze out is of order

$$
n_{F} \sim\left(M_{N} T_{F}\right)^{3 / 2} e^{-M_{N} / T_{F}} .
$$

From this we find that $T_{F} \lesssim M_{N} / 15$, for all $M_{N}$ below $100 \mathrm{GeV}$. This shows that the number density of relic composite singlet neutrinos is exponentially suppressed at temperatures below the compositeness scale. However, as we shall see later, these may still give rise to observable effects.

\subsection{Cosmological bounds on the compositeness scale}

Cosmological observations can be used to place constraints on the compositeness scale $\Lambda$. There are two effects that we need to consider.

- The sizable self-interactions that arise from the partial compositeness of neutrinos can prevent the neutrinos from free streaming during the CMB era. This is disfavored by CMB measurements, leading to a lower bound on $\Lambda$.

- The CMB also places bounds on the total energy density in radiation during the epoch of acoustic oscillations. These can be translated into a lower bound on the compositeness scale $\Lambda$.

We now consider these effects in turn.

Limits on neutrino free streaming. Precision measurements of the CMB have established that the neutrinos are free streaming during the epoch of acoustic oscillations [94-96]. This can be used to place limits on the size of neutrino self-interactions. In particular, the mean free path of the neutrinos must be greater than the size of the universe at temperatures of order an eV. In our framework, the cross section for elastic neutrino-neutrino scattering at temperatures below the compositeness scale $\Lambda$ is of order,

$$
\sigma_{\nu \nu \rightarrow \nu \nu} \sim \frac{\kappa^{2}}{4 \pi}\left(\frac{\lambda v_{\mathrm{EW}}}{m_{N}}\right)^{8} \frac{T^{2}}{\Lambda^{4}}
$$

Then the inverse of the mean free path for neutrino scattering is of order,

$$
n_{\nu} \sigma_{\nu \nu \rightarrow \nu \nu} \sim \frac{\kappa^{2}}{4 \pi}\left(\frac{\lambda v_{\mathrm{EW}}}{m_{N}}\right)^{8} \frac{T^{5}}{\Lambda^{4}} \gtrsim \frac{\kappa^{2}}{4 \pi}\left(\frac{m_{\nu}}{m_{N}}\right)^{4} \frac{T^{5}}{\Lambda^{4}},
$$


where the inequality is obtained by setting $\lambda$ to its lower bound in eq. (2.18). To satisfy the constraint on free streaming this must be less than the Hubble scale, $H=g_{*} T^{2} / M_{\mathrm{Pl}}$, at temperatures of order an $\mathrm{eV}$. This translates into a lower bound on the compositeness scale in this scenario, $\Lambda \gtrsim 100 \mathrm{eV}$.

Limits on the energy density in radiation. The predictions of Big Bang Nucleosynthesis (BBN) in the SM are in excellent agreement with data [98]. This can be used to place a strong limit on any additional energy density in radiation from physics beyond the $\mathrm{SM}$ at temperatures of order an $\mathrm{MeV}$, the time at which the neutrinos decouple from the rest of the SM. This bound implies that the hidden sector cannot be in equilibrium with the SM at temperatures of order an $\mathrm{MeV}$. This constraint is automatically satisfied if the compositeness scale lies above an $\mathrm{MeV}$, so that the hadrons of the strongly coupled sector exit the bath prior to BBN. In principle, the bound can also be satisfied if the compositeness scale lies below an $\mathrm{MeV}$, provided that the hidden sector is initially at a lower temperature than the SM and comes into equilibrium with the neutrinos only after BBN.

There are also strong limits on the total energy density in radiation during the epoch of acoustic oscillations based on precision measurements of the CMB [97]. The current upper bound stands at $N_{\text {eff }} \lesssim 3.5$. As we now explain, this constraint disfavors compositeness scales $\Lambda$ less than an $\mathrm{MeV}$, even in the case that the hidden sector comes into equilibrium with the neutrinos only at temperatures below an MeV. The SM neutrinos decouple from the rest of the SM bath at temperatures of order an $\mathrm{MeV}$. If the compositeness scale lies below an $\mathrm{MeV}$, then the only SM particles that the composite sector will be in equilibrium with at temperatures of order $\Lambda$ are the neutrinos. When the strongly coupled sector comes into equilibrium with the neutrinos, the comoving energy density is conserved, while the comoving entropy density increases [99-101]. However, when the composite states go out of the bath, the comoving entropy density is conserved, while the comoving energy density increases. Therefore, if the compositeness scale lies below an $\mathrm{MeV}$, we expect an increase in the total comoving energy density in the light neutrinos at the time of CMB over the SM prediction. The size of this effect depends on the number of degrees of freedom in the hidden sector. Unless this number is very small (less than the equivalent of 2 Weyl fermion degrees of freedom), this class of theories is disfavored by the current CMB bound on $N_{\text {eff }}$. We therefore focus our attention on the regime $\Lambda \gtrsim 1 \mathrm{MeV}$.

\subsection{Spectral distortions of the CMB from late decays}

Through their mixing with the SM neutrinos, the relic composite singlet neutrinos eventually decay through the weak interactions into final states composed of SM particles. The decay width of the singlet neutrinos can be estimated from eq. (3.13),

$$
\Gamma_{N} \sim \frac{G_{F}^{2}}{192 \pi^{3}}\left(\frac{\lambda v_{\mathrm{EW}}}{M_{N}}\right)^{2} M_{N}^{5} \gtrsim \frac{G_{F}^{2} M_{N}^{4}}{192 \pi^{3}} m_{\nu}
$$

where the inequality is obtained by setting $\lambda$ to its lower bound in eq. (2.18). For $M_{N} \lesssim$ $1 \mathrm{GeV}$ the composite singlet neutrinos decay when the temperature of the SM bath is below an $\mathrm{MeV}$. This could potentially affect the successful predictions of BBN by dissociating 
nuclei. However, our calculation of the relic abundance of the composite singlet neutrinos shows that their number density is too small to have an observable effect on BBN.

For $M_{N} \lesssim 50 \mathrm{MeV}$ and $\lambda$ set to its minimum value, the relic singlet neutrinos decay at even later times, when the temperature of the bath is at or below a keV. One of the primary decay channels is $N \rightarrow e^{+} e^{-} \nu$. The resulting injection of electromagnetic energy into the bath at these late times gives rise to spectral distortions in the CMB. Although photon-number conserving processes are still active at these low temperatures, photonnumber changing processes such as double Compton scattering are comparatively slow. Any injection of electromagnetic energy at this time will manifest itself in a $\mu$-distortion of the CMB spectrum. ${ }^{3}$ For an electromagnetic decay fraction $\mathfrak{f}$, the shift in $\mu$ is approximately given by $[102,103]$,

$$
\mu \approx 8 \times 10^{2}\left(\frac{\tau_{N}}{1 \mathrm{sec}}\right)^{1 / 2}\left(\frac{m_{N}}{\mathrm{GeV}}\right)\left[\frac{n_{N}\left(\tau_{N}\right)}{n_{\gamma}\left(\tau_{N}\right)}\right] g\left(\frac{\tau_{\mathrm{dC}}}{\tau_{N}}\right) \mathfrak{f},
$$

with

$$
g(x) \approx\left\{\begin{array}{ll}
e^{-x^{5 / 4}} & x \lesssim 1 \\
2.1 x^{5 / 9} e^{-2 x^{5 / 9}} & x \gtrsim 1
\end{array} .\right.
$$

Here the double Compton time scale is given by $\tau_{\mathrm{dC}} \approx 7 \times 10^{6}$ sec. Since $N \rightarrow e^{+} e^{-} \nu$ is one of the primary decay modes, $\mathfrak{f} \sim 1$. For $\Lambda \lesssim 50 \mathrm{MeV}$, these effects can be as large as $\mu \sim 10^{-8}$. Although consistent with current observations, a spectral distortion of this size is large enough to be seen at next generation CMB experiments such as PIXIE [104]. For a given value of $M_{N}$, increasing $\lambda$ above its lower bound in eq. (2.18) will hasten the decay of the composite singlet neutrinos and reduce the $\mu$-distortion. Therefore, in contrast to the other signals we have studied, spectral distortions are more sensitive to lower values of the mixing angle.

\section{Astrophysics}

We now turn our attention to the limits on the compositeness scale $\Lambda$ from astrophysical considerations. We expect that the effects of neutrino compositeness are only important in astrophysical scenarios where either the temperature or the density are comparable to $\Lambda$. Since the cosmological bounds already constrain $\Lambda \gtrsim 1 \mathrm{MeV}$, which is many orders of magnitude higher than the temperature in a typical star, we expect that neutrino compositeness will not have a significant impact on stellar dynamics. However, the temperature $T_{\mathrm{SN}}$ at the core of a supernova is of order $40 \mathrm{MeV}$. Then, if the compositeness scale is low enough, composite singlet neutrinos can be produced in the core, and could potentially affect the supernova dynamics. In particular, the emission of composite singlet neutrinos now offers a new mechanism for the core to lose energy. If this energy loss occurs too rapidly, the explosion cannot take place. In the remainder of this section, we investigate this question

\footnotetext{
${ }^{3}$ Due to the letter $\mu$ has been taken as a Lagrangian parameter in this model, here we use $\mu$ to represent the $\mu$-distortion of the CMB spectrum.
} 
further. We will first consider the regime $\Lambda \gg T_{\mathrm{SN}}$, corresponding to a compositeness scale well above the supernova temperature, and discuss the limit $\Lambda \lesssim T_{\mathrm{SN}}$ later.

We begin by determining the conditions under which any composite singlet neutrinos that are produced are able to escape freely from the supernova. The scattering processes $\nu N \rightarrow N N$ and $\nu N \rightarrow \nu N$ act to prevent the composite neutrinos from escaping. The requirement that singlet neutrinos are trapped in the supernova by the $\nu N \rightarrow N N$ process corresponds to the condition,

$$
n_{\nu}\left\langle\sigma_{\nu N \rightarrow N N}\right\rangle L_{\mathrm{SN}} \gg 1
$$

where $L_{\mathrm{SN}} \sim 10 \mathrm{~km}$ is the size of the supernova and $n_{\nu}$, the number density of neutrinos inside the core, can be estimated as $T_{\mathrm{SN}}^{3}$. The corresponding condition in the case of the $\nu N \rightarrow \nu N$ process takes the form,

$$
n_{\nu}\left\langle\sigma_{\nu N \rightarrow \nu N}\right\rangle L_{\mathrm{SN}} \gg 1
$$

The inverse of the mean free path for the process $\nu N \rightarrow N N$ at a temperature $T_{\mathrm{SN}}$ can be estimated as,

$$
n_{\nu}\left\langle\sigma_{\nu N \rightarrow N N}\right\rangle \approx \frac{\kappa^{2}}{4 \pi}\left(\frac{\lambda v_{\mathrm{EW}}}{M_{N}}\right)^{2} \frac{M_{N}^{2} T_{\mathrm{SN}}^{3}}{\Lambda^{4}} \exp \left(-M_{N} / T_{\mathrm{SN}}\right)
$$

where the exponential suppression arises because we are in the region of parameter space where the mass splitting between the initial and final states is greater than the temperature in the supernova core. Setting $\lambda$ to its lower bound in eq. (2.18), we find that this process prevents the composite singlet neutrinos from escaping provided that the compositeness scale $\Lambda \lesssim 800 \mathrm{MeV}$. Conversely, if we set $\lambda$ to its upper bound in eq. (2.18), we find that the composite singlet neutrinos remain trapped in the supernova for all compositeness scales $\Lambda \lesssim 1.7 \mathrm{GeV}$. Turning our attention to the process $\nu N \rightarrow \nu N$, the inverse of the mean free path can be estimated as,

$$
n_{\nu}\left\langle\sigma_{\nu N \rightarrow \nu N}\right\rangle \approx \frac{\kappa^{2}}{4 \pi}\left(\frac{\lambda v_{\mathrm{EW}}}{M_{N}}\right)^{4} \frac{M_{N}^{2} T_{\mathrm{SN}}^{3}}{\Lambda^{4}}
$$

For $\lambda$ at its lower bound, we find that this process prevents the composite singlet neutrinos from escaping for all $\Lambda \lesssim 300 \mathrm{MeV}$. For $\lambda$ at its upper bound, the corresponding limit on $\Lambda$ is $10^{9} \mathrm{GeV}$.

It follows from this discussion that for all $\Lambda$ below $800 \mathrm{MeV}$, the composite singlet neutrinos will be trapped in the supernova by the $\nu N \rightarrow N N$ process. For larger values of $\Lambda$, the composite singlet neutrinos may still be prevented from escaping but only if the coupling $\lambda$ is large enough. For smaller couplings of $\lambda$ any composite singlet neutrinos that are produced will free stream out of the supernova.

The scattering process $\nu N \rightarrow N N$ has the effect of increasing the number density of composite singlet neutrinos in the supernova. Therefore, in the regime that eq. (8.1) is satisfied, their number density will increase exponentially quickly provided that a few seed composite singlet neutrinos are initially present. This will have the effect of bringing the composite singlet neutrinos into thermal and chemical equilibrium inside the core 
of the supernova. However, because their equilibrium number density is suppressed as $\exp \left(-M_{N} / T_{\mathrm{SN}}\right)$, we expect that their impact on supernova dynamics is limited. If eq. (8.1) is not satisfied, the $N$-number changing process is slow. However, for large enough $\lambda$, eq. (8.2) may be satisfied so that the composite singlet neutrinos are trapped by the $\nu N \rightarrow \nu N$ process. In this regime, the composite singlet neutrinos would reach kinetic but not chemical equilibrium. For smaller values of $\lambda$, neither eq. (8.1) nor eq. (8.2) is satisfied, and any composite singlet neutrinos that are produced will free stream out of the supernova. However, as we now argue, in this regime their production rate is exponentially suppressed and the resulting energy loss is too small to affect the supernova dynamics.

There are two processes that seed the initial production of composite singlet neutrinos inside the supernova, $\nu \nu \rightarrow N N$ and $\nu \nu \rightarrow N \nu$. The first process requires the total energy in the incoming neutrinos to be at least $2 M_{N}$ but the amplitude is suppressed by only two powers of the $\nu-N$ mixing. For the second process, the total energy in the initial state particles need only be $M_{N}$, but the amplitude is now suppressed by an additional power of $\nu-N$ mixing. The production of the seed $N$ states must occur on the timescales shorter than the lifetime of a supernova $\left(t_{\mathrm{SN}} \sim 10 \mathrm{sec}\right)$. The number of seed composite singlet neutrinos produced through the process $\nu \nu \rightarrow N N$ over the lifetime of the supernova can be estimated as,

$$
\frac{\kappa^{2}}{4 \pi}\left(\frac{\lambda v_{\mathrm{EW}}}{M_{N}}\right)^{4} \frac{M_{N}^{2}}{\Lambda^{4}} e^{-2 M_{N} / T_{\mathrm{SN}}}\left(T_{\mathrm{SN}}^{2} L_{\mathrm{SN}}\right)^{3} t_{\mathrm{SN}} .
$$

Setting $\lambda$ to its lower bound in eq. (2.18), we find that at least a few composite singlet neutrinos are produced in the supernova through this process provided $\Lambda \lesssim 2.5 \mathrm{GeV}$. If $\lambda$ is at its upper bound, the corresponding upper bound on the compositeness scale is $\Lambda \lesssim$ $3 \mathrm{GeV}$. Turning our attention to the process $\nu \nu \rightarrow N \nu$, the number of seed composite singlet neutrinos produced can be estimated as,

$$
\frac{\kappa^{2}}{4 \pi}\left(\frac{\lambda v_{\mathrm{EW}}}{M_{N}}\right)^{6} \frac{M_{N}^{2}}{\Lambda^{4}} e^{-M_{N} / T_{\mathrm{SN}}}\left(T_{\mathrm{SN}}^{2} L_{\mathrm{SN}}\right)^{3} t_{\mathrm{SN}}
$$

For $\lambda$ at its lower bound in eq. (2.18), we find that at least a few seed composite singlet neutrinos will be produced through this process in the supernova core on the relevant timescale provided $\Lambda \lesssim 4 \mathrm{GeV}$. For $\lambda$ at its upper bound, the corresponding limit is $\Lambda \lesssim 5 \mathrm{GeV}$.

In summary, when $\lambda$ is at its lower bound in eq. (2.18), some number of composite singlet neutrinos are produced inside the supernova core provided $\Lambda \lesssim 4 \mathrm{GeV}$. For $\Lambda \lesssim$ $800 \mathrm{MeV}$, these particles are trapped inside the supernova, and come into thermal and chemical equilibrium. For $\Lambda$ in the range $800 \mathrm{MeV} \lesssim \Lambda \lesssim 4 \mathrm{GeV}$, the composite singlet neutrinos are able to stream freely out of the supernova. However, from eqs. (8.5) and (8.6), we find that the resulting energy loss is too small to affect the supernova dynamics. Turning to the limit when $\lambda$ is at its upper bound in eq. (2.18), some number of composite singlet neutrinos are produced inside the supernova core provided $\Lambda \lesssim 5 \mathrm{GeV}$. Once produced, these particles remain trapped inside the core. For $\Lambda \lesssim 1.7 \mathrm{GeV}$, they come into thermal and chemical equilibrium. For values of $\Lambda$ above $1.7 \mathrm{GeV}$ they enter into thermal equilibrium but not chemical equilibrium. For intermediate values of $\lambda$ the dynamics is 
expected to lie between these two extremes. We conclude that for $\Lambda \gg T_{\mathrm{SN}}$, there is no significant contribution to supernova cooling from the hidden sector.

We now turn our attention to the case when the compositeness scale is less than or comparable to the supernova temperature, $\Lambda \lesssim T_{\mathrm{SN}}$. In this regime composite singlet neutrinos are easily produced, and once produced, will be trapped inside the supernova. Now however, since $T_{\mathrm{SN}} \gtrsim \Lambda$, once the trapped singlet neutrinos approach thermal equilibrium with the SM inside the supernova, the composite dynamics may undergo a phase transition back to the conformal phase. If the number of degrees of freedom in the hidden sector is sizable, this could potentially have a large impact on the supernova dynamics. However, given that at present our understanding of supernova explosions is limited, it is difficult to use this to place a robust constraint.

Our discussion up till now has not taken into account the effects of the chemical potential of the electron neutrinos in the supernova core. This is of order $200 \mathrm{MeV}$, higher then $T_{\mathrm{SN}}$. The primary effect of the chemical potential is to make the supernova even more opaque to composite singlet neutrinos, and so the conclusion that there is no significant contribution to supernova cooling from the hidden sector remains unaltered. Now however, some part of the chemical potential could be passed on to the trapped composite singlet neutrinos, increasing their net abundance inside the supernova core. For compositeness scales of order $200 \mathrm{MeV}$ or lower, this effect may be large enough to significantly affect the supernova dynamics. However, it is once again not clear that this leads to a robust constraint. We defer a careful study of the effects of this class of models on supernovae for future work.

\section{Conclusions}

We have proposed a framework in which the SM neutrinos mix with the composite fermions of a strongly coupled hidden sector through the neutrino portal. The light neutrinos are then partially composite particles. An explicit breaking of lepton number in the hidden sector allows the neutrinos to obtain small Majorana masses. From the low energy perspective this framework leads to an inverse seesaw model for neutrino masses in which the masses of the singlet neutrinos are set by the compositeness scale. However, if probed at energies above the compositeness scale, where the constituents of the singlet neutrinos are strongly coupled, the phenomenology of this class of models differs greatly from that of a conventional inverse seesaw. For example, the decay of SM particles to the hidden sector can now result in multiple singlet neutrinos rather than just one. In addition, the large selfinteractions of the composite singlet neutrinos allow them to annihilate away efficiently in the early universe, so that the severe cosmological bounds on singlet neutrinos with masses below a $\mathrm{GeV}$ are naturally satisfied.

We have outlined a specific realization of this scenario in which, in the ultraviolet, the composite sector is a strongly coupled CFT. The small parameters necessary to obtain realistic neutrino masses in the framework of a low-scale seesaw naturally arise as a consequence of the scaling dimensions of operators in the CFT. Neutrino masses could be small either because the mixing with composite states is dynamically suppressed, or 
because lepton number is an approximate symmetry of the hidden sector at the compositeness scale, with a continuum of possibilities between these two extremes. The approximate lepton number symmetry could also arise as a consequence of dynamical effects. This gives a concrete but very broad range of possibilities for the compositeness scale and for phenomenology. In particular, the scale of neutrino compositeness can be as low as an MeV. Below an $\mathrm{MeV}$, we find that the cosmological limits on $N_{\text {eff }}$ disfavor this scenario.

We have shown that this class of models has important implications for a wide range of experiments, including colliders and beam dumps, searches for lepton flavor violation and neutrinoless double beta decay, and precision observations of the CMB spectral shape. SM particles can now decay to final states that include multiple composite singlet neutrinos. These particles can be long-lived, resulting in striking displaced vertex signals at colliders and beam dumps. At loop level, the composite sector can contribute to lepton flavor violation processes such as $\mu \rightarrow e \gamma$ and $\mu \rightarrow e$ conversion at rates that can be probed in upcoming experiments such as COMET and Mu2e. If the compositeness scale is of order the pion mass or below, the rate of neutrinoless double beta decay will be suppressed by form factors. For compositeness scales below $50 \mathrm{MeV}$, the late decays of relic composite singlet neutrinos can give rise to spectral distortions in the CMB that are large enough to be observed in next generation experiments.

\section{Acknowledgments}

ZC and ZL are supported in part by the National Science Foundation under Grant Number PHY-1914731. ZC is also supported in part by the US-Israeli BSF Grant 2018236. ZL is also supported in part by the Maryland Center for Fundamental Physics. ZC would like to thank the Fermilab Theory Group for hospitality during the completion of this work. ZC's stay at Fermilab was supported by the Fermilab Intensity Frontier Fellowship and the Visiting Scholars Award \#17-S-02 from the Universities Research Association. PF, RH, and ZL would like to thank the Aspen Center for Physics which is supported by National Science Foundation grant PHY-1607611, where part of the study was performed. PF and $\mathrm{RH}$ would like to thank the Galileo Galilei Institute for hospitality where part of this work was completed, and PF would like to thank the Simons Foundation (SIMONS, 341344 AL) for support. PF and RH are supported by Fermi Research Alliance, LLC under Contract DE-AC02-07CH11359 with the U.S. Dept. of Energy.

\section{A Matrix elements and widths}

For the process $W \rightarrow \ell \mathcal{U}$, the matrix element is,

$$
i \mathcal{M}=\frac{g}{\sqrt{2}} \frac{\hat{\lambda} v_{\mathrm{EW}}}{M_{\mathrm{UV}}^{\Delta_{\mathrm{N}}-3 / 2}} \bar{\ell} \gamma^{\mu} \frac{1}{2}\left(1-\gamma_{5}\right) \frac{\not \mathrm{X}}{N^{2}} N \epsilon_{\mu}(W),
$$

where $g$ is the $\mathrm{SU}(2)$ gauge coupling. $\bar{\ell}$ and $N$ are the spinors of the charged lepton and the unparticle state, and $\epsilon_{\mu}(W)$ is the polarization vector of the $W$ boson with $W$ being its 4-momenta. 
In evaluation of the spin and polarization averaged matrix element squared, we use the identity,

$$
\sum_{\text {pols. }} \epsilon_{\mu} \epsilon_{\nu}^{*}=-g_{\mu \nu}+\frac{W_{\mu} W_{\nu}}{m_{W}^{2}}
$$

and we obtain,

$$
\begin{aligned}
|\overline{\mathcal{M}}|^{2} & =g^{2} \frac{\left|\hat{\lambda} v_{\mathrm{EW}}\right|^{2}}{M_{\mathrm{UV}}^{2 \Delta_{\mathrm{N}}-3} m_{N}^{2}}(\ell \cdot N)\left(2+\frac{m_{N}^{2}}{m_{W}^{2}}\right) \\
& =g^{2} \frac{\left|\hat{\lambda} v_{\mathrm{EW}}\right|^{2}}{2 M_{\mathrm{UV}}^{2 \Delta_{\mathrm{N}}-3} m_{N}^{2}}\left(m_{W}^{2}-m_{N}^{2}\right)\left(2+\frac{m_{N}^{2}}{m_{W}^{2}}\right)
\end{aligned}
$$

where $\ell$ and $N$ are the 4-momenta of the corresponding states, and we have ignored the mass of $\ell$. In the $W$ rest frame $m_{N}^{2}=m_{W}^{2}-2 m_{W} E_{\ell}$ and the matrix element squared becomes,

$$
|\overline{\mathcal{M}}|^{2}=g^{2} \frac{\left|\hat{\lambda} v_{\mathrm{EW}}\right|^{2}}{2 M_{\mathrm{UV}}^{2 \Delta_{\mathrm{N}}-3}} \frac{E_{\ell}}{m_{W}} \frac{3 m_{W}-2 E_{\ell}}{m_{W}-2 E_{\ell}} .
$$

The only kinematic quantity this matrix element depends on is the lepton energy $E_{\ell}$. Using the rest frame relation $p_{N}^{2}=m_{W}^{2}-2 m_{W} E_{\ell}$, the integration over lepton and unparticle phase space can be carried out,

$$
\begin{aligned}
\Gamma= & \int_{0}^{1} \frac{g^{2} m_{W}}{192 \pi^{2}} \frac{\left|\hat{\lambda} v_{\mathrm{EW}}\right|^{2}}{m_{W}^{2}}\left(\frac{m_{W}}{M}\right)^{2 \Delta_{\mathrm{N}}-3} A_{\Delta_{\mathrm{N}}-1 / 2}\left(\frac{3 m_{W}-2 E_{\ell}}{m_{W}-2 E_{\ell}}\right)\left(\frac{2 E_{\ell}}{m_{W}}\right)^{2} \\
& \times\left(1-\frac{2 E_{\ell}}{m_{W}}-\frac{\mu_{\mathrm{IR}}^{2}}{m_{W}^{2}}\right)^{\Delta_{\mathrm{N}}-5 / 2} \theta\left(1-\frac{2 E_{\ell}}{m_{W}}-\frac{\mu_{\mathrm{IR}}^{2}}{m_{W}^{2}}\right) d \frac{2 E_{\ell}}{m_{W}} \\
= & \frac{g^{2} m_{W}}{192 \pi^{2}} \frac{\left|\hat{\lambda} v_{\mathrm{EW}}\right|^{2}}{m_{W}^{2}}\left(\frac{m_{W}}{M}\right)^{2 \Delta_{\mathrm{N}}-3} A_{\Delta_{\mathrm{N}}-1 / 2} \times f\left(\Delta_{\mathrm{N}}, \delta\right),
\end{aligned}
$$

where, we have defined $\delta \equiv \mu_{\mathrm{IR}}^{2} / m_{W}^{2}$. The full expression for $f\left(\Delta_{\mathrm{N}}, \delta\right)$ is cumbersome,

$$
\begin{aligned}
f\left(\Delta_{\mathrm{N}}, \delta\right)= & -4(1-\delta)^{\Delta_{\mathrm{N}}-\frac{3}{2}} \frac{4\left(\Delta_{\mathrm{N}}-1\right)\left(\Delta_{\mathrm{N}}+2\right)-2(2 \delta-1)(\delta-1)-4 \delta \Delta_{\mathrm{N}}+7}{\left(2 \Delta_{\mathrm{N}}-3\right)\left(2 \Delta_{\mathrm{N}}-1\right)\left(2 \Delta_{\mathrm{N}}+1\right)} \\
& +2 \delta^{\Delta_{\mathrm{N}}-\frac{5}{2}} B_{\frac{1}{\delta}-1}\left(\Delta_{\mathrm{N}}-\frac{5}{2}\right)
\end{aligned}
$$

where $B_{z}(a)$ is

$$
B_{z}(a)=\int_{0}^{z} d t \frac{t^{a}}{1+t} .
$$

Some interesting limits for the product of $f\left(\Delta_{\mathrm{N}}, \delta\right)$ and the phase volume factor $A_{\Delta_{\mathrm{N}}-1 / 2}$ are

$$
\begin{aligned}
\lim _{\Delta_{\mathrm{N}} \rightarrow \frac{3}{2}} A_{\Delta_{\mathrm{N}}-\frac{1}{2}} f\left(\Delta_{\mathrm{N}}, \delta\right) & =\frac{2 \pi\left(\delta^{3}-3 \delta+2\right)}{\delta} \\
\lim _{\delta \rightarrow 0} A_{\Delta_{\mathrm{N}}-\frac{1}{2}} f\left(\Delta_{\mathrm{N}}, \delta\right) & =\left(16 \pi^{2}\right)^{2-\Delta_{\mathrm{N}}} \frac{\Gamma\left(\frac{5}{2}-\Delta_{\mathrm{N}}\right)}{\Gamma\left(\Delta_{\mathrm{N}}-\frac{1}{2}\right)} \delta^{\Delta_{\mathrm{N}}-\frac{5}{2}}+\mathcal{O}\left(\delta^{0}\right) .
\end{aligned}
$$


When the infrared cutoff $\mu_{\mathrm{IR}}$ in the unparticle treatment is identified with the mass of the composite singlet neutrinos $M_{N}$, our calculations agree with the standard results for elementary HNLs in the $\Delta_{\mathrm{N}} \rightarrow 3 / 2$ limit.

For meson decays, neglecting the lepton mass, the partial width is given by

$$
\begin{aligned}
d \Gamma(\mathfrak{m} \rightarrow \ell \mathcal{U})= & \frac{G_{F}^{2}\left|V_{q q^{\prime}}\right|^{2} f_{\mathfrak{m}}^{2}\left|\hat{\lambda} v_{\mathrm{EW}}\right|^{2}}{M_{\mathrm{UV}}^{2 \Delta_{\mathrm{N}}-3}}\left(2 E_{\ell}\right) d \Phi_{\left(\Delta_{\mathrm{N}}-1 / 2\right)+1} \\
= & \frac{m_{\mathfrak{m}} G_{F}^{2}\left|V_{q q^{\prime}}\right|^{2} f_{\mathfrak{m}}^{2}\left|\hat{\lambda} v_{\mathrm{EW}}\right|^{2}}{32 \pi^{2}}\left(\frac{m_{\mathfrak{m}}}{M_{\mathrm{UV}}}\right)^{2 \Delta_{\mathrm{N}}-3} A_{\Delta_{\mathrm{N}}-1 / 2}\left(\frac{2 E_{e}}{m_{\mathfrak{m}}}\right)^{2} \\
& \times\left(1-\frac{2 E_{\ell}}{m_{\mathfrak{m}}}-\frac{\mu_{\mathrm{IR}}^{2}}{m_{\mathfrak{m}}^{2}}\right)^{\Delta_{\mathrm{N}}-5 / 2} \theta\left(1-\frac{2 E_{\ell}}{m_{\mathfrak{m}}}-\frac{\mu_{\mathrm{IR}}^{2}}{m_{\mathfrak{m}}^{2}}\right) d \frac{2 E_{\ell}}{m_{\mathfrak{m}}}
\end{aligned}
$$

where $m_{\mathfrak{m}}$ is the mass of the charged meson.

After integration, the partial width of $\mathfrak{m} \rightarrow e \mathcal{U}$ is,

$$
\Gamma(\mathfrak{m} \rightarrow e \mathcal{U})=\frac{m_{\mathfrak{m}} G_{F}^{2}\left|V_{q q^{\prime}}\right|^{2} f_{\mathfrak{m}}^{2}\left|\hat{\lambda} v_{\mathrm{EW}}\right|^{2}}{32 \pi^{2}}\left(\frac{m_{\mathfrak{m}}}{M_{\mathrm{UV}}}\right)^{2 \Delta_{\mathrm{N}}-3} A_{\Delta_{\mathrm{N}}-1 / 2} \times g\left(\Delta_{\mathrm{N}}, \frac{\mu_{\mathrm{IR}}^{2}}{m_{\mathfrak{m}}^{2}}\right)
$$

with

$$
g\left(\Delta_{\mathrm{N}}, \delta\right)=\frac{16(1-\delta)^{\Delta_{N}+\frac{1}{2}}}{\left(2 \Delta_{\mathrm{N}}-3\right)\left(2 \Delta_{\mathrm{N}}-1\right)\left(2 \Delta_{\mathrm{N}}+1\right)} .
$$

In this case the two limits of interest are,

$$
\begin{aligned}
\lim _{\Delta_{\mathrm{N}} \rightarrow \frac{3}{2}} A_{\Delta_{\mathrm{N}}-\frac{1}{2}} g\left(\Delta_{\mathrm{N}}, \delta\right) & =2 \pi(1-\delta)^{2}, \\
\lim _{\delta \rightarrow 0} A_{\Delta_{\mathrm{N}}-\frac{1}{2}} g\left(\Delta_{\mathrm{N}}, \delta\right) & =\frac{\left(16 \pi^{2}\right)^{2-\Delta_{\mathrm{N}}}}{\Gamma\left(\Delta_{\mathrm{N}}+\frac{3}{2}\right) \Gamma\left(\Delta_{\mathrm{N}}-\frac{1}{2}\right)}+\mathcal{O}(\delta) .
\end{aligned}
$$

Open Access. This article is distributed under the terms of the Creative Commons Attribution License (CC-BY 4.0), which permits any use, distribution and reproduction in any medium, provided the original author(s) and source are credited.

\section{References}

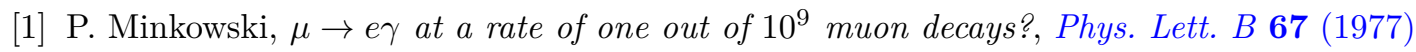
421 [INSPIRE].

[2] T. Yanagida, Horizontal symmetry and masses of neutrinos, in the proceedings of the Workshop on unified theory and baryon number in the universe, O. Sawada and A. Sugamoto eds., KEK, Tsukuba, Japan (1979).

[3] M. Gell-Mann, P. Ramond and R. Slansky, Complex Spinors and Unified Theories, Conf. Proc. C $\mathbf{7 9 0 9 2 7}$ (1979) 315 [arXiv: 1306.4669] [InSPIRE].

[4] S.L. Glashow, The future of elementary particle physics, in Quarks and leptons, Cargèse lectures, M. Lévy et al. eds., Plenum Press, New York, U.S.A. (1980).

[5] R.N. Mohapatra and G. Senjanović, Neutrino mass and spontaneous parity nonconservation, Phys. Rev. Lett. 44 (1980) 912 [INSPIRE]. 
[6] W. Konetschny and W. Kummer, Nonconservation of total lepton number with scalar bosons, Phys. Lett. B 70 (1977) 433 [InSPIRE].

[7] M. Magg and C. Wetterich, Neutrino mass problem and gauge hierarchy, Phys. Lett. B 94 (1980) 61 [INSPIRE].

[8] J. Schechter and J.W.F. Valle, Neutrino masses in $\mathrm{SU}(2) \times \mathrm{U}(1)$ theories, Phys. Rev. D 22 (1980) 2227 [INSPIRE].

[9] T.P. Cheng and L.-F. Li, Neutrino masses, mixings and oscillations in $\mathrm{SU}(2) \times \mathrm{U}(1)$ models of electroweak interactions, Phys. Rev. D 22 (1980) 2860 [InSPIRE].

[10] G. Lazarides, Q. Shafi and C. Wetterich, Proton lifetime and fermion masses in an $\mathrm{SO}(10)$ model, Nucl. Phys. B 181 (1981) 287 [InSPIRE].

[11] R.N. Mohapatra and G. Senjanović, Neutrino masses and mixings in gauge models with spontaneous parity violation, Phys. Rev. D 23 (1981) 165 [INSPIRE].

[12] R. Foot, H. Lew, X.G. He and G.C. Joshi, Seesaw neutrino masses induced by a triplet of leptons, Z. Phys. C 44 (1989) 441 [inSPIRE].

[13] E. Ma, Pathways to naturally small neutrino masses, Phys. Rev. Lett. 81 (1998) 1171 [hep-ph/9805219] [INSPIRE].

[14] G.B. Gelmini and M. Roncadelli, Left-handed neutrino mass scale and spontaneously broken lepton number, Phys. Lett. B 99 (1981) 411 [INSPIRE].

[15] Y. Chikashige, R.N. Mohapatra and R.D. Peccei, Are there real goldstone bosons associated with broken lepton number?, Phys. Lett. B 98 (1981) 265 [INSPIRE].

[16] H.M. Georgi, S.L. Glashow and S. Nussinov, Unconventional model of neutrino masses, Nucl. Phys. B 193 (1981) 297 [InSPIRE].

[17] N. Arkani-Hamed and Y. Grossman, Light active and sterile neutrinos from compositeness, Phys. Lett. B 459 (1999) 179 [hep-ph/9806223] [INSPIRE].

[18] G. von Gersdorff and M. Quirós, Conformal neutrinos: an alternative to the see-saw mechanism, Phys. Lett. B 678 (2009) 317 [arXiv:0901.0006] [INSPIRE].

[19] Y. Grossman and D.J. Robinson, Composite Dirac neutrinos, JHEP 01 (2011) 132 [arXiv: 1009.2781] [INSPIRE].

[20] D.J. Robinson and Y. Tsai, KeV warm dark matter and composite neutrinos, JHEP 08 (2012) 161 [arXiv: 1205.0569] [INSPIRE].

[21] D.J. Robinson and Y. Tsai, Dynamical framework for KeV Dirac neutrino warm dark matter, Phys. Rev. D 90 (2014) 045030 [arXiv:1404.7118] [INSPIRE].

[22] R.S. Hundi and S. Roy, Constraints on composite Dirac neutrinos from observations of galaxy clusters, Phys. Lett. B 702 (2011) 228 [arXiv:1105.0291] [INSPIRE].

[23] Y. Grossman and Y. Tsai, Leptogenesis with composite neutrinos, JHEP 12 (2008) 016 [arXiv: 0811.0871] [INSPIRE].

[24] T. Okui, Searching for composite neutrinos in the cosmic microwave background, JHEP 09 (2005) 017 [hep-ph/0405083] [INSPIRE].

[25] R.N. Mohapatra, Mechanism for understanding small neutrino mass in superstring theories, Phys. Rev. Lett. 56 (1986) 561 [INSPIRE].

[26] R.N. Mohapatra and J.W.F. Valle, Neutrino mass and baryon number nonconservation in superstring models, Phys. Rev. D 34 (1986) 1642 [INSPIRE]. 
[27] B. Bellazzini, C. Csáki and J. Serra, Composite Higgses, Eur. Phys. J. C 74 (2014) 2766 [arXiv: 1401.2457] [INSPIRE].

[28] G. Panico and A. Wulzer, The composite nambu-Goldstone higgs, Springer, Germany (2016) [arXiv: 1506.01961] [INSPIRE].

[29] D.B. Kaplan, Flavor at SSC energies: a new mechanism for dynamically generated fermion masses, Nucl. Phys. B 365 (1991) 259 [inSPIRE].

[30] R. Contino and A. Pomarol, Holography for fermions, JHEP 11 (2004) 058 [hep-th/0406257] [INSPIRE].

[31] H. Georgi, Unparticle physics, Phys. Rev. Lett. 98 (2007) 221601 [hep-ph/0703260] [INSPIRE].

[32] H. Georgi, Another odd thing about unparticle physics, Phys. Lett. B 650 (2007) 275 [arXiv: 0704 .2457] [INSPIRE].

[33] J.M. Maldacena, The large $N$ limit of superconformal field theories and supergravity, Int. $J$. Theor. Phys. 38 (1999) 1113 [hep-th/9711200] [Adv. Theor. Math. Phys. 2 (1998) 231] [INSPIRE].

[34] S.S. Gubser, I.R. Klebanov and A.M. Polyakov, Gauge theory correlators from noncritical string theory, Phys. Lett. B 428 (1998) 105 [hep-th/9802109] [INSPIRE].

[35] E. Witten, Anti-de Sitter space and holography, Adv. Theor. Math. Phys. 2 (1998) 253 [hep-th/9802150] [INSPIRE].

[36] I.R. Klebanov and E. Witten, AdS/CFT correspondence and symmetry breaking, Nucl. Phys. B 556 (1999) 89 [hep-th/9905104] [InSPIRE].

[37] N. Arkani-Hamed, M. Porrati and L. Randall, Holography and phenomenology, JHEP 08 (2001) 017 [hep-th/0012148] [inSPIRE].

[38] R. Rattazzi and A. Zaffaroni, Comments on the holographic picture of the Randall-Sundrum model, JHEP 04 (2001) 021 [hep-th/0012248] [INSPIRE].

[39] L. Randall and R. Sundrum, A large mass hierarchy from a small extra dimension, Phys. Rev. Lett. 83 (1999) 3370 [hep-ph/9905221] [INSPIRE].

[40] Y. Grossman and M. Neubert, Neutrino masses and mixings in nonfactorizable geometry, Phys. Lett. B 474 (2000) 361 [hep-ph/9912408] [INSPIRE].

[41] S.J. Huber and Q. Shafi, Majorana neutrinos in a warped $5-D$ standard model, Phys. Lett. $B \mathbf{5 4 4}$ (2002) 295 [hep-ph/0205327] [InSPIRE].

[42] S.J. Huber and Q. Shafi, Seesaw mechanism in warped geometry, Phys. Lett. B 583 (2004) 293 [hep-ph/0309252] [INSPIRE].

[43] T. Gherghetta, Dirac neutrino masses with Planck scale lepton number violation, Phys. Rev. Lett. 92 (2004) 161601 [hep-ph/0312392] [INSPIRE].

[44] K. Agashe, S. Hong and L. Vecchi, Warped seesaw mechanism is physically inverted, Phys. Rev. D 94 (2016) 013001 [arXiv: 1512.06742] [INSPIRE].

[45] K.L. McDonald, Light neutrinos from a mini-seesaw mechanism in warped space, Phys. Lett. B 696 (2011) 266 [arXiv:1010.2659] [INSPIRE].

[46] B. Gripaios, Neutrinos in a sterile throat, Nucl. Phys. B 768 (2007) 157 [Erratum ibid. 830 (2010) 390] [hep-ph/0611218] [INSPIRE].

[47] G. Cacciapaglia, G. Marandella and J. Terning, Colored unparticles, JHEP 01 (2008) 070 [arXiv:0708.0005] [INSPIRE]. 
[48] S. Weinberg, Phenomenological lagrangians, Physica A 96 (1979) 327 [InSPIRE].

[49] A. Manohar and H. Georgi, Chiral quarks and the nonrelativistic quark model, Nucl. Phys. B 234 (1984) 189 [INSPIRE].

[50] H. Georgi and L. Randall, Flavor conserving CP-violation in invisible axion models, Nucl. Phys. B 276 (1986) 241 [INSPIRE].

[51] M. Kenzie, A review of unparticle physics, arXiv:0809.5092.

[52] A. Atre, T. Han, S. Pascoli and B. Zhang, The search for heavy Majorana neutrinos, JHEP 05 (2009) 030 [arXiv:0901.3589] [INSPIRE].

[53] CMS collaboration, Search for heavy neutral leptons in events with three charged leptons in proton-proton collisions at $\sqrt{s}=13$ TeV, Phys. Rev. Lett. 120 (2018) 221801 [arXiv: 1802.02965] [INSPIRE].

[54] ATLAS collaboration, Search for heavy neutral leptons in decays of $W$ bosons produced in $13 \mathrm{TeV}$ pp collisions using prompt and displaced signatures with the ATLAS detector, JHEP 10 (2019) 265 [arXiv: 1905.09787] [INSPIRE].

[55] DELPHI collaboration, Search for neutral heavy leptons produced in $Z$ decays, Z. Phys. $C$ 74 (1997) 57 [Erratum ibid. 75 (1997) 580] [INSPIRE].

[56] J. Liu, Z. Liu, L.-T. Wang and X.-P. Wang, Seeking for sterile neutrinos with displaced leptons at the LHC, JHEP 07 (2019) 159 [arXiv: 1904.01020] [INSPIRE].

[57] CMS collaboration, Search for displaced leptons in the e-mu channel, CMS-PAS-EXO-16-022 (2016).

[58] M. Drewes and J. Hajer, Heavy Neutrinos in displaced vertex searches at the LHC and HL-LHC, JHEP 02 (2020) 070 [arXiv: 1903.06100] [INSPIRE].

[59] E.J. Chun, A. Das, S. Mandal, M. Mitra and N. Sinha, Sensitivity of lepton number violating meson decays in different experiments, Phys. Rev. D 100 (2019) 095022 [arXiv:1908.09562] [INSPIRE].

[60] J. Beacham et al., Physics beyond colliders at CERN: beyond the standard model working group report, J. Phys. G 47 (2020) 010501 [arXiv: 1901.09966] [INSPIRE].

[61] G. Cottin, J.C. Helo and M. Hirsch, Displaced vertices as probes of sterile neutrino mixing at the LHC, Phys. Rev. D 98 (2018) 035012 [arXiv:1806.05191] [INSPIRE].

[62] Neutrino non-standard interactions: a status report, in the proceedings of the NTN Workshop on Neutrino Non-Standard Interactions, may 29-31, St. Louis, U.S.A. (2019) [INSPIRE].

[63] K. Bondarenko, A. Boyarsky, D. Gorbunov and O. Ruchayskiy, Phenomenology of GeV-scale heavy neutral leptons, JHEP 11 (2018) 032 [arXiv:1805.08567] [INSPIRE].

[64] M.J. Strassler and K.M. Zurek, Echoes of a hidden valley at hadron colliders, Phys. Lett. B 651 (2007) 374 [hep-ph/0604261] [inSPIRE].

[65] T. Han, Z. Si, K.M. Zurek and M.J. Strassler, Phenomenology of hidden valleys at hadron colliders, JHEP 07 (2008) 008 [arXiv:0712.2041] [INSPIRE].

[66] P. Schwaller, D. Stolarski and A. Weiler, Emerging jets, JHEP 05 (2015) 059 [arXiv: 1502.05409] [INSPIRE].

[67] A. Pierce, B. Shakya, Y. Tsai and Y. Zhao, Searching for confining hidden valleys at LHCb, ATLAS, and CMS, Phys. Rev. D 97 (2018) 095033 [arXiv:1708.05389] [inSPIRE]. 
[68] J. Alimena et al., Searching for long-lived particles beyond the Standard Model at the Large Hadron Collider, J. Phys. G 47 (2020) 090501 [arXiv: 1903. 04497] [InSPIRE].

[69] C. Yuan, H. Zhang and Y. Zhao, Producing and detecting long-lived particles at different experiments at the LHC, arXiv:2004.08820 [INSPIRE].

[70] D.I. Britton et al., Improved search for massive neutrinos in $\pi^{+} \rightarrow e^{+}$neutrino decay, Phys. Rev. D 46 (1992) 885 [InSPIRE].

[71] PIENU collaboration, Improved search for heavy neutrinos in the decay $\pi \rightarrow e \nu$, Phys. Rev. D 97 (2018) 072012 [arXiv: 1712.03275] [INSPIRE].

[72] Annecy-CERn-London(IC)-Paris(LPNHE) collaboration, Proposal: search for decays of heavy neutrinos with the PS beam, CERN-PSCC-83-127 (1983).

[73] CHARM collaboration, A search for decays of heavy neutrinos in the mass range $0.5 \mathrm{GeV}$ to $2.8 \mathrm{GeV}$, Phys. Lett. B 166 (1986) 473 [INSPIRE].

[74] NA62 collaboration, Search for heavy neutral lepton production in $K^{+}$decays to positrons, Phys. Lett. B 807 (2020) 135599 [arXiv: 2005.09575] [INSPIRE].

[75] T2K collaboration, Search for heavy neutrinos with the T2K near detector ND280, Phys. Rev. D 100 (2019) 052006 [arXiv: 1902.07598] [INSPIRE].

[76] S. Alekhin et al., A facility to search for hidden particles at the CERN SPS: the SHiP physics case, Rept. Prog. Phys. 79 (2016) 124201 [arXiv:1504.04855] [InSPIRE].

[77] LBNE collaboration, The long-baseline neutrino experiment: exploring fundamental symmetries of the universe, talk given at Snowmass 2013: Workshop on Energy Frontier, July 20-August 6, U.S.A. (2013) [arXiv:1307.7335] [INSPIRE].

[78] T. Blum et al., The muon $(g-2)$ theory value: present and future, arXiv:1311.2198 [INSPIRE].

[79] M. Lindner, M. Platscher and F.S. Queiroz, A call for new physics: the muon anomalous magnetic moment and lepton flavor violation, Phys. Rept. 731 (2018) 1 [arXiv: 1610.06587] [INSPIRE].

[80] Muon G-2 collaboration, Muon $(g-2)$ technical design report, arXiv:1501.06858 [INSPIRE].

[81] COMET collaboration, COMET Phase-I technical design report, PTEP 2020 (2020) 033C01 [arXiv: 1812.09018] [INSPIRE].

[82] Mu2E collaboration, Mu2e technical design report, arXiv:1501.05241 [INSPIRE].

[83] S.R. Moore, K. Whisnant and B.-L. Young, Second order corrections to the muon anomalous magnetic moment in alternative electroweak models, Phys. Rev. D 31 (1985) 105 [INSPIRE].

[84] P.J. Fox, A. Rajaraman and Y. Shirman, Bounds on unparticles from the Higgs sector, Phys. Rev. D 76 (2007) 075004 [arXiv:0705.3092] [INSPIRE].

[85] F. Jegerlehner, Muon g-2 theory: The hadronic part, EPJ Web Conf. 166 (2018) 00022 [arXiv: 1705.00263] [INSPIRE].

[86] MEG collaboration, Search for the lepton flavour violating decay $\mu^{+} \rightarrow \mathrm{e}^{+} \gamma$ with the full dataset of the MEG experiment, Eur. Phys. J. C 76 (2016) 434 [arXiv:1605.05081] [INSPIRE].

[87] R. Alonso, M. Dhen, M.B. Gavela and T. Hambye, Muon conversion to electron in nuclei in type-I seesaw models, JHEP 01 (2013) 118 [arXiv:1209.2679] [INSPIRE]. 
[88] SINDRUM II collaboration, A search for muon to electron conversion in muonic gold, Eur. Phys. J. C $\mathbf{4 7}$ (2006) 337 [inSPIRE].

[89] Mu2E collaboration, The Mu2e Experiment, Front. in Phys. 7 (2019) 1 [arXiv: 1901.11099] [INSPIRE].

[90] J. Lopez-Pavon, S. Pascoli and C.-f. Wong, Can heavy neutrinos dominate neutrinoless double beta decay?, Phys. Rev. D 87 (2013) 093007 [arXiv: 1209.5342] [INSPIRE].

[91] P.D. Bolton, F.F. Deppisch and P.S. Bhupal Dev, Neutrinoless double beta decay versus other probes of heavy sterile neutrinos, JHEP 03 (2020) 170 [arXiv:1912.03058] [INSPIRE].

[92] I.T. Todorov, M.C. Mintchev and V.B. Petkova, Conformal invariance in quantum field theory, Edizioni della Normale, Italy (1978).

[93] E. Fradkin and M. Palchik, Conformal quantum field theory in D-dimensions, Springer, Germany (1996).

[94] M. Archidiacono and S. Hannestad, Updated constraints on non-standard neutrino interactions from Planck, JCAP 07 (2014) 046 [arXiv:1311.3873] [INSPIRE].

[95] F. Forastieri, M. Lattanzi and P. Natoli, Constraints on secret neutrino interactions after Planck, JCAP 07 (2015) 014 [arXiv: 1504.04999] [INSPIRE].

[96] N. Blinov and G. Marques-Tavares, Interacting radiation after Planck and its implications for the Hubble tension, JCAP 09 (2020) 029 [arXiv:2003.08387] [INSPIRE].

[97] Planck collaboration, Planck 2018 results. VI. Cosmological parameters, Astron. Astrophys. 641 (2020) A6 [arXiv:1807.06209] [INSPIRE].

[98] R.H. Cyburt, B.D. Fields, K.A. Olive and T.-H. Yeh, Big Bang nucleosynthesis: 2015, Rev. Mod. Phys. 88 (2016) 015004 [arXiv: 1505.01076] [InSPIRE].

[99] Z. Chacko, L.J. Hall, T. Okui and S.J. Oliver, CMB signals of neutrino mass generation, Phys. Rev. D 70 (2004) 085008 [hep-ph/0312267] [InSPIRE].

[100] Z. Chacko, L.J. Hall, S.J. Oliver and M. Perelstein, Late time neutrino masses, the LSND experiment and the cosmic microwave background, Phys. Rev. Lett. 94 (2005) 111801 [hep-ph/0405067] [INSPIRE].

[101] A. Berlin and N. Blinov, Thermal dark matter below an MeV, Phys. Rev. Lett. 120 (2018) 021801 [arXiv: 1706.07046] [INSPIRE].

[102] W. Hu and J. Silk, Thermalization constraints and spectral distortions for massive unstable relic particles, Phys. Rev. Lett. 70 (1993) 2661 [INSPIRE].

[103] J. Chluba and R.A. Sunyaev, The evolution of CMB spectral distortions in the early Universe, Mon. Not. Roy. Astron. Soc. 419 (2012) 1294 [arXiv:1109.6552] [INSPIRE].

[104] A. Kogut et al., The Primordial Inflation Explorer (PIXIE): a nulling polarimeter for Cosmic Microwave Background observations, JCAP 07 (2011) 025 [arXiv:1105.2044] [INSPIRE]. 